\title{
Quantum Kinetic Theory V: Quantum kinetic master equation for mutual interaction of condensate and noncondensate
}

\author{
C.W. Gardiner ${ }^{1}$ and P. Zoller ${ }^{2}$ \\ ${ }^{1}$ Physics Department, Victoria University, Wellington, New Zealand \\ ${ }^{2}$ Insitut für Theoretische Physik, Universität Innsbruck, 6020 Innsbruck, Austria
}

\begin{abstract}
A detailed quantum kinetic master equation is developed which couples the kinetics of a trapped condensate to the vapor of non-condensed particles. This generalizes previous workwhich treated the vapor as being undepleted.
\end{abstract}

\section{INTRODUCTION}

Until the experimental realization of a Bose-Einstein condensate of magnetically trapped Alkali atoms [1-3] in 1995 (which has now become a widely available technology [4]) the theory of Bose-Einstein condensation was dominated by the desire to understand the behavior of superfluid liquid helium, of which it was assumed that the weakly interacting degenerate Bose gas was a simplified and admittedly inadequate model. The central tools for the description of BEC were the Bogoliubov theory of superfluidity and modifications of it [5]7], the Gross-Pitaevskii equation [8] and techniques based on the Green's function formalism of many body theory [9]. This philosophy is excellently presented in Griffin's book [10].

The prospect of actually producing a degenerate Bose gas gave rise to a number of investigations [19] based largely on a combination of kinetic theory as described by the UehlingUhlenbeck equation [18] and the the Gross-Pitaevskii equation. However, nearly all of these investigations were from the point of view of macroscopic statistical mechanics, in which the BEC was seen as a sample of an extended fluid, rather than the tightly trapped condensate, which behaves more like a very large atom or molecule than a droplet of macroscopic fluid.

Our own program of quantum kinetic theory has been formulated in such a way as to take advantage the simplifications which can be made for a tightly trapped condensate. It has been partially presented in our first four papers on the subject [11], as well as in two papers on the growth of a Bose-Einstein condensates [16,17]. However, all of the previous papers covered only special situations. In QKI we treated only spatially homogeneous systems with at most a small amount of condensate; QKII dealt with stochastic equations as a model of the initiation of the condensate, QKIII and QKIV considered only the case where the majority of the atoms provided a bath of atoms with energy above a certain value (called $E_{R}$ ) of fixed chemical potential $\mu$ and temperature $T$ for a relatively small number of lower energy atoms, called the condensate band, which included the condesnsate level itself as well as the levels with energy less than $E_{R}$.

In this paper we will present what we see as a comprehensive and reasonably practical description of the subject, in which the various restrictions in the previous papers are largely eliminated. The description which results is best described in terms of a system composed of a condensate and a vapor in interaction with each other. The vapor is described by a quantum kinetic master equation of the form introduced in QKI, but appropriately adapted to take account of the presence of a trap. For nearly all situations, this is equivalent to a quantum Boltzmann equation of the Uehling-Uhlenbeck [18] form. The condensate is described by a fully quantum mechanical Hamiltonian, which for many purposes can be treated by a Bogoliubov approximation, but truncated to include only those excitations not already included in the vapor. The interaction between vapor and condensate consists of two parts; a Hamiltonian mean field term, and a master equation term which describes the transfer of energy and particles between vapor and condensate. The largest part of the mean field term can be estimated, and included by defining effective potentials for the condensate and the vapor. The computation of these effective potentials involves a procedure very similar to that of the Hartree-Fock-Bogoliubov [20] method. However these effective potentials are used only to give a basis set of eigenfunctions, in terms of which we can describe the kinetics; the resulting kinetic equations give rise to corrections to the corresponding eigenvalues-our use of a basis of eigenfunctions similar to those given by the Hartree-FockBogoliubov method does not mean our method is the same as the that method, since there is a residual term remaining after the majority of the mean field term has been included in the effective potentials, and this is of a similar order of magnitude to the master equation term.

This paper is concerned mainly with methodology and reasonably careful derivations, rather than with directly applicable results. However, it provides a justification for the heuristic description of the theory of condensate growth presented in [17], and does provide a way in which hydrodynamics and quantum kinetics can be connected together. There are, of course alternative approaches to the general issue of combining kinetic theory and quantum mechanics, which have different emphases. For example Stoof [21] has chosen to develop an approach which avoids the use of the pseudopotential method, which he has shown will fail very near the critical point; Walser et al. [22] have developed a kinetic approach somewaht more related to ours; while Zaremba et al. 23. have developed a two fluid hydrodynamic description. The major criterion for choosing a particular approach will ultimately be 
the degree of usefulness in explaining experiments, and its ease of use-but we will leave this judgment on this to the BEC community.

In the remainder of this introduction we will summarize the philosophy and scope of our methodology, and to emphasize the most significant results.

\section{A. The field theory of Bose particles}

We want to consider the Bose atoms to be described by a second-quantized field, in the pseudopotential approximation; that is, we write

$$
H=H_{\mathrm{kin}}+H_{I}+H_{T},
$$

where

$$
\begin{aligned}
H_{\mathrm{kin}} & =\int d^{3} \mathbf{x} \psi^{\dagger}(\mathbf{x})\left(-\frac{\hbar^{2}}{2 m} \nabla^{2}\right) \psi(\mathbf{x}), \\
H_{I} & =\frac{1}{2} \int d^{3} \mathbf{x} \int d^{3} \mathbf{x}^{\prime} \psi^{\dagger}(\mathbf{x}) \psi^{\dagger}\left(\mathbf{x}^{\prime}\right) u\left(\mathbf{x}-\mathbf{x}^{\prime}\right) \psi\left(\mathbf{x}^{\prime}\right) \psi(\mathbf{x})
\end{aligned}
$$

and the term $H_{T}$ arises from a trapping potential as

$$
H_{T}=\int d^{3} \mathbf{x} V_{T}(\mathbf{x}) \psi^{\dagger}(\mathbf{x}) \psi(\mathbf{x})
$$

In this paper we will not go deeply into the approximations which allow us to use the pseudopotential form of the Hamiltonian, in which

$$
u\left(\mathbf{x}^{\prime}-\mathbf{x}\right) \rightarrow u \delta\left(\mathbf{x}^{\prime}-\mathbf{x}\right)=\frac{4 \pi a}{m} \delta\left(\mathbf{x}^{\prime}-\mathbf{x}\right),
$$

which is thoroughly investigated in many works [24]. Suffice it to say only that this is an approximation whose validity is assured provided the wavelengths of the atoms are very much longer than the range of the potential $u\left(\mathbf{x}^{\prime}-\mathbf{x}\right)$, and provided that we are not working too close to the critical point. Thus, at typical temperatures and densities currently used, this approximation is valid either when there is a significant amount of condensate, or when there is no condensate. The essence of the derivation of this pseudopotential approximation is really an adiabatic elimination of the fast motion of the atomic wavefunctions during the very small proportion of time during which they are actually undergoing collisions. Thus the result is effectively a Hamiltonian which is coarse-grained in time, and thus, because of the dispersion relation, also in space. The details of this philosophy are available in a number of references [24] Having made this approximation, we know we are then justified in using an approach which is essentially perturbative.

\section{B. Thermalization assumptions}

It is evident in experiments that a typical condensate in a trap is accompanied by a thermal vapor cloud. The condensate consists of a large number of particles, all of which share the same wavefunction, while the vapor is essentially completely thermalized - it thus consists of particles which do not share wavefunctions with each other, and indeed most wavefunctions are not occupied by any particles at all. The vapor consists mainly of the higher energy particles, but there is a transition from vapor to condensate which, althought not sharp, necessitates the drawing of a definited boundary between them.
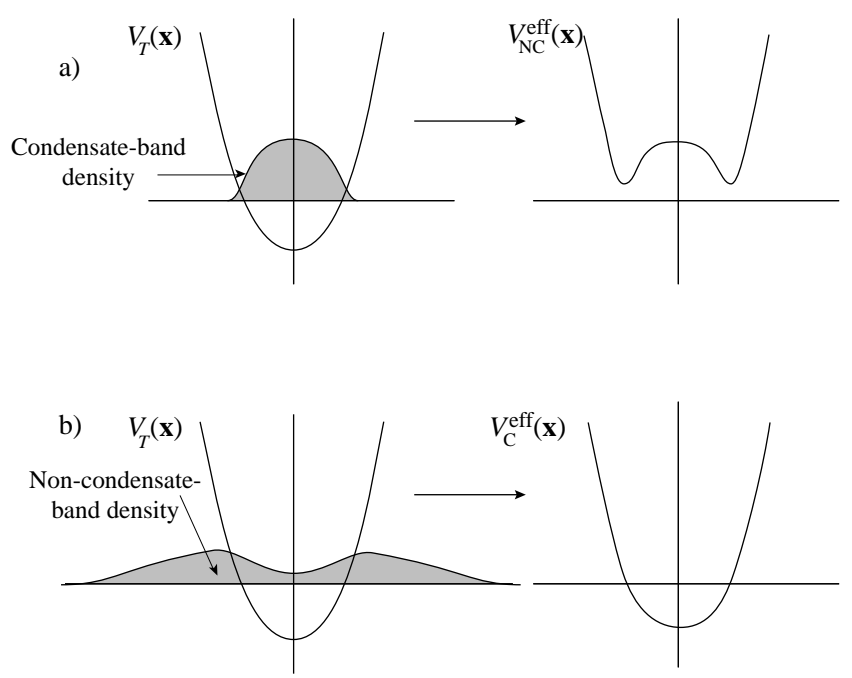

Fig. 1: Representation of the modification of the trapping potential for a) the non-condensate band and b) the condensate band due to mean-field effects

\section{Condensate and non-condensate bands in the previous papers}

The concept of condensate and non-condensate bands was introduced by us in QKIII and [16]. In these earlier works we chose to make the distinction between these in terms of the effect of the presence of a condensate on the trap energy levels. This meant that the non-condensate band was taken to consist of those energy levels above a certain value, $E_{R}$, which were treated as being essentially unaffected by the condensate; the condensate band consisted of all levels with energies $E<E_{R}$, and these were taken to have wavefunctions and energies which were given by the Bogoliubov method.

In this paper we move to a more useful way of treating the two bands. In QKI we developed a wavelet description of weakly condensed systems, which allowed for a fully quantum-mechanical description of the thermalization, but this was derived only for the case of free particles. It was the absence of a good wavelet description of trapped particles that led us to consider in QKIII only the approximation that the non-condensate band could be treated as being like a bath of thermal particles with a fixed chemical potential, $\mu$ and a fixed temperature, $T$. Attention was then concentrated on the derivation QKIII and successful application [16, 15, 17] of a master equation for the condensate band. 


\section{Condensate and non-condensate bands in this paper}

In this paper we have worked out how the wavelet description can be modified in the presence of a trapping potential, and have been able to give a full set of coupled equations connecting the two bands. The distinction between the two bands is now made in terms of whether the energy level is particle like, or phonon-like. We take the division between the bands to be at $E_{R}$, where $E_{R}$ is the energy above which all the excitations can be considered, to a good degree of approximation, to consist of a definite number of particles, unlike the phononlike excitations, which have lower energies.

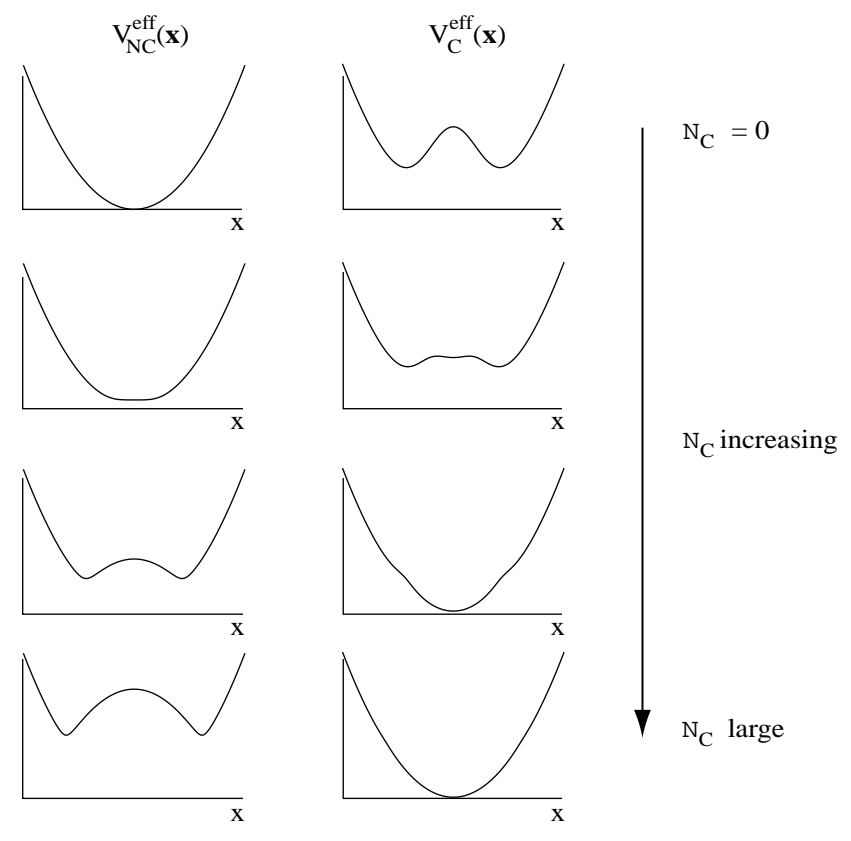

Fig. 2: Representation of the non-condensate-band (left) and condensate band (right) effective potentials appropriate to an increasing number of condensate atoms. Fore each particular sector, defined by the numbers $N_{\mathrm{NC}}$ and $N_{\mathrm{C}}$, the different effective potentials define the basis eigenstates in terms of which the master equation is defined. The process of growth is that of transition from one sector to the next, and does not involve a time dependence of the potential.

In order to take account of mean-field effects of the condensate-band atoms on the non-condensate-band atoms, and conversely, we use effective trap potentials, for the condensate and non-condensate bands which include these meanfield effects. Thus, we write

$$
\begin{aligned}
V_{\mathrm{NC}}^{\mathrm{mf}}(\mathbf{x}) & =2 u[\bar{\rho}(\mathbf{x})+\bar{n}(\mathbf{x})], \\
V_{\mathrm{C}}^{\mathrm{mf}}(\mathbf{x}) & =2 u \bar{n}(\mathbf{x})
\end{aligned}
$$

where $\bar{\rho}(\mathbf{x})$ is the mean condensate band density, and $\bar{n}(\mathbf{x})$ is the mean density of the vapor in the non-condensate band. The values of these quantities depend on the particular physical situation, including the numbers of particles, $N_{\mathrm{NC}}$ and $N_{\mathrm{C}}$, in the respective bands.

The effective potentials are then

$$
\begin{aligned}
& V_{\mathrm{NC}}^{\mathrm{eff}}(\mathbf{x})=V_{T}(\mathbf{x})+V_{\mathrm{NC}}^{\mathrm{mf}}(\mathbf{x}) \\
& V_{\mathrm{C}}^{\mathrm{eff}}(\mathbf{x})=V_{T}(\mathbf{x})+V_{\mathrm{C}}^{\mathrm{mf}}(\mathbf{x})
\end{aligned}
$$

As written, the effective potentials contain no timedependence. However, time-dependent situations can be dealt with in two ways.

Firstly, consider condensate growth. The picture put forward by us in [16, 17] treats condensate growth as the slow change of the populations of the condensate and noncondensate bands by transitions of particles from one band to the other. The transitions are between energy levels determined by the values of the densities $\bar{n}(\mathbf{x}), \bar{\rho}(\mathbf{x})$ appropriate to the actual numbers $N_{\mathrm{NC}}, N_{\mathrm{C}}$ of non-condensate-band and condensate-band atoms. Thus the values of $\bar{n}(\mathbf{x})$ and $\bar{\rho}(\mathbf{x})$ (and hence also the mean-field ptentials) will change adiabatically as the condensate grows, and the energy levels needed to compute the transition rates will also change adiabatically. The basic requirement is that the growth is sufficiently slow compared to the enrgy thermalization time within each band that there are always well-defined values of $\bar{n}(\mathbf{x})$ and $\bar{\rho}(\mathbf{x})$.

The second way in which time-dependence can be treated is perturbatively. If the condensate and vapor oscillate with small amplitude about mean values, we can show that this can be taken into account by using appropriate time dependent values of $\bar{n}(\mathbf{x})$ and $\bar{\rho}(\mathbf{x})$.

These two effective potentials provide a basis within which we can develop the full quantum kinetic theory. This is done by dividing the field operator into two parts

$$
\psi(\mathbf{x})=\phi(\mathbf{x})+\psi_{\mathrm{NC}}(\mathbf{x})
$$

which then correspond to the condensate band and noncondensate band degrees of freedom. We then write the Hamiltonian (1.1) as

$$
H=H^{\mathrm{eff}}+\left(H-H^{\mathrm{eff}}\right)
$$

in which $H^{\text {eff }}$ is the sum of the condensate-band and noncondensate band effective Hamiltonians; hence the noncondensate-band effective Hamiltonian is

$$
H_{\mathrm{NC}}^{\mathrm{eff}}=\int d^{3} \mathbf{x} \psi^{\dagger}(\mathbf{x})\left\{-\frac{\hbar^{2} \nabla^{2}}{2 m}+V_{\mathrm{NC}}^{\mathrm{eff}}(\mathbf{x})\right\} \psi(\mathbf{x})
$$

and the condensate band effective Hamiltonian is

$$
\begin{aligned}
H_{\mathrm{C}}^{\mathrm{eff}}= & \int d^{3} \mathbf{x} \phi^{\dagger}(\mathbf{x})\left\{-\frac{\hbar^{2} \nabla^{2}}{2 m}+V_{\mathrm{C}}^{\mathrm{eff}}(\mathbf{x})\right\} \\
& +\frac{u}{2} \int d^{3} \mathbf{x} \phi^{\dagger}(\mathbf{x}) \phi^{\dagger}(\mathbf{x}) \phi(\mathbf{x}) \phi(\mathbf{x}) .
\end{aligned}
$$

The quantities $\bar{\rho}(\mathbf{x})$ and $\bar{n}(\mathbf{x})$ are nominally the mean condensate-band and non-condensate-band densities, but the real criterion for their choice is to minimize the effective interaction term $H-H^{\text {eff }}$ 


\section{Basis states for the master equation}

The non-condensate-band effective Hamiltonian is now written in terms of a wavelet basis, as follows. We write

$$
\psi_{\mathrm{NC}}(\mathbf{x})=\sum_{\mathbf{Q}, \mathbf{r}} w_{\mathbf{Q}}(\mathbf{x}, \mathbf{r}) A_{\mathbf{Q}, \mathbf{r}} \equiv \sum_{\mathbf{Q}} \psi_{\mathbf{Q}}(\mathbf{x})
$$

Here $A_{\mathbf{Q}, \mathbf{r}}$ are destruction operators with the commutation relations

$$
\left[A_{\mathbf{Q}, \mathbf{r}}, A_{\mathbf{Q}^{\prime}, \mathbf{r}^{\prime}}^{\dagger}\right]=\delta_{\mathbf{Q Q}^{\prime}} \delta_{\mathbf{r r}^{\prime}}
$$

so that $\mathbf{r}$ and $\mathbf{Q}$ are discrete indices. The wavelets $w_{\mathbf{Q}}(\mathbf{x}, \mathbf{r})$ are a complete orthonormal set, such that

1. $w_{\mathbf{Q}}(\mathbf{x}, \mathbf{r}) \rightarrow 0$ when $|\mathbf{r}-\mathbf{x}|$ is large.

2. $w_{\mathbf{Q}}(\mathbf{x}, \mathbf{r})$ is a linear combination of trap eigenfunctions for the effective potential $V_{T}(\mathbf{x})+V_{\mathrm{NC}}^{\mathrm{mf}}(\mathbf{r})$ with energy eigenvalues in a range restricted to a neighborhood of $\hbar^{2} \mathbf{Q}^{2} / 2 m$.

3. With each set of $\mathbf{Q}, \mathbf{r}$ we can associate a momentum $\hbar \mathbf{K}(\mathbf{Q}, \mathbf{r})$ which is such that

$$
\frac{\hbar^{2} \mathbf{Q}^{2}}{2 m}=\frac{\hbar^{2} \mathbf{K}^{2}}{2 m}+V_{\mathrm{NC}}^{\mathrm{eff}}(\mathbf{r}) .
$$

For every direction of $\mathrm{Q}$ we can also associate a direction of $\mathbf{K}$; for example in a spherically symmetric harmonic trap $\mathbf{Q}$ and $\mathbf{K}$ are parallel.

In general a rule of association between the directions is not simple, but it can be specified implicitly.

4. Using the function $\mathbf{K}(\mathbf{Q}, \mathbf{r})$ we can write the commutator

$$
\left[\psi_{\mathbf{Q}}(\mathbf{x}), \psi_{\mathbf{Q}^{\prime}}^{\dagger}\left(\mathbf{x}^{\prime}\right)\right] \approx \delta_{\mathbf{Q} \mathbf{Q}^{\prime}} e^{i \mathbf{K}\left(\mathbf{Q}, \frac{\mathbf{x}+\mathbf{x}^{\prime}}{2}\right) \cdot\left(\mathbf{x}-\mathbf{x}^{\prime}\right)} g_{\mathbf{Q}}\left(\mathbf{x}, \mathbf{x}^{\prime}\right)
$$

where the function $g_{\mathbf{Q}}\left(\mathbf{x}, \mathbf{x}^{\prime}\right)$ is only significantly different from zero when $\mathrm{x} \approx \mathrm{x}^{\prime}$, and is normalized to one in both variables $\mathbf{x}$ and $\mathbf{x}^{\prime}$.

This set of wavelets can be explicitly constructed in a number of cases, and we assume its existence in general. It provides a resolution of the non-condensate field into discrete "cells" in phases space labeled by $\mathbf{Q}$ and $r$. The wavelet function $w_{\mathbf{Q}, \mathbf{r}}(\mathbf{x})$ corresponds to a particle approximately localized at $\mathbf{r}$ in space and with momentum in a small range around $\hbar \mathbf{K}(\mathbf{Q}, \mathbf{r})$. Its total energy is thus approximately $E(\mathbf{Q}) \equiv \hbar^{2} \mathbf{Q}^{2} / 2 m$, which is independent of $\mathbf{r}$. A particle in a wavelet state $\mathbf{Q}, \mathbf{r}$ will evolve under the influence of the Hamiltonian $H_{\mathrm{NC}}^{\mathrm{eff}}$ into a linear combination of wavelets with the same $\mathbf{Q}$, so that the evolution represents energy conserving and particle conserving flow in phase space. We thus obtain a Liouvillian flow in this discrete phase space.

The evolution of the condensate band is described by (1.13), which differs from the full Hamiltonian only in that

1. The mean-field potential of the vapor $V_{\mathrm{C}}^{\mathrm{mf}}(\mathbf{x})$ occurs.
2. The operator $\phi(\mathbf{x})$ is limited to the lower energy levels of the full Hamiltonian, and thus $\phi(\mathbf{x})$ has a non-local commutation relation, the degree of non-locality being of the same size as the average spatial width of the wavelets used to construct the non-condensate-band.

Although we have described the wavelets by approximate properties, once the wavelets have been constructed, the description in terms of them is exact. However, in order to use this description some approximations are still necessary. The simplest procedure - and the one we shall use here-is to use the number-conserving Bogoliubov model, but include the vapor mean-field potential $V_{\mathrm{C}}^{\mathrm{mf}}(\mathbf{x})=2 u \bar{n}(\mathbf{x})$ as well as the trap potential. This provides us with an approximate resolution of the non-condensate-band field operator in the form

$$
\phi(\mathbf{x})=A\left(\xi_{N_{\mathrm{C}}}(\mathbf{x})+\frac{1}{\sqrt{N_{\mathrm{C}}+1}} \chi(\mathbf{x})\right)
$$

in which

1. $A$ is a destruction operator for the total number of atoms in the condensate band;

2. $N_{\mathrm{C}} \equiv A^{\dagger} A$ is the number of atoms in the condensate band;

3. $\xi_{N_{\mathrm{C}}}(\mathbf{x})$ is the condensate wavefunction.

4. The quantity $\chi(\mathbf{x})$ is a quasiparticle field operator, which has the expansion

$$
\chi(\mathbf{x})=\sum_{m}\left\{p_{m}(\mathbf{x}) b_{m}+q_{m}(\mathbf{x}) b_{m}^{\dagger}\right\} .
$$

The $p_{m}(\mathbf{x})$ and $q_{m}(\mathbf{x})$ are quasiparticle amplitudes for the quasiparticle creation and destruction operators $b_{m}^{\dagger}, b_{m}$. The condensate has energy $\mu\left(N_{\mathrm{C}}\right)$, and each quasiparticles carries an energy $\hbar \epsilon_{m}\left(N_{\mathrm{C}}\right)$.

We assume that $N_{\mathrm{C}}$ is almost equal to the number of particles $N$ in the condensate level. This is a significantly weaker requirement than that which would be necessary to use the usual Bogoliubov method for the whole system, which would require the total number of particles in the system, $N_{\mathrm{C}}+N_{\mathrm{NC}}$, to be close to the number of particles in the condensate.

At this degree of approximation, the description is rather like the conventional Hartree-Fock-Bogoliubov [20] method. However, there is so far no thermalization assumed-this can only be done satisfactorily by introducing irreversibility, which we shall do using our master equation.

\section{The master equation}

The master equation has two main purposes

1. To take account of the interactions that are included in the term $H-H^{\text {eff }}$ in the equation (1.12)

\section{To introduce irreversibility, and thus thermalization.}

The technical methodology we use is very similar to that used in QKI. The equation of motion for the density operator is developed perturbatively by projecting onto the space 
of density operators in which the relative phases of noncondensate-band states with different $\mathbf{Q}$ are eliminated. We cannot eliminate the phases corresponding to the same $\mathbf{Q}$ and different $\mathbf{r}$, since the Liouvillian transport generated by $H^{\text {eff }}$ is off-diagonal in $\mathbf{r}$, would itself be eliminated by such a procedure. But Liouvillian transport generates the streaming terms in the quantum Boltzmann equation, and must therefore be preserved if we are to obtain a full hydrodynamic description of the vapor.

As in QKI and QKIII the derivation of the master equation relies on a perturbation expansion in the interaction term, and on a random phase approximation in certain of the coefficients. The master equation which results is natural, selfconsistent and consistent with statistical mechanics.

For example, a stationary solution for the density operator can be written

$$
\rho_{s}=\exp \left(-\frac{H_{a}^{\mathrm{eff}}-\mu N_{\mathrm{NC}}}{k T}\right) \otimes \exp \left(-\frac{H_{\mathrm{C}}^{\mathrm{eff}}-\mu N_{\mathrm{C}}}{k T}\right) .
$$

Here

$$
H_{a}^{\mathrm{eff}}=\sum_{\mathbf{r}, \mathbf{Q}} E(\mathbf{Q}) A_{\mathbf{Q}, \mathbf{r}}^{\dagger} A_{\mathbf{Q}, \mathbf{r}}
$$

and $H_{\mathrm{C}}^{\mathrm{eff}}$ is given by 1.13 .

There are thus no correlations between the condensate and non-condensate bands, and none between the different $\mathbf{Q}, \mathbf{r}$ "cells" in the non-condensate-band. This description is selfconsistent if the highest energy states in the condensate-band (and all higher energy states) are essentially particle-like, since the density operator will then look much the same at the top of the condensate-band as at the bottom of the noncondensate band. The master equation in the full form is written in Sect.IIIE, and comprises terms which account for all physical processes as follows.

1. Condensate-band term: This is given by $3.35,3.37$, and arises from the condensate band Hamiltonian $H_{\mathrm{C}}^{\mathrm{eff}}, 1.13$ ).

2. Non-condensate-band terms: These are given by $\sqrt{3.38 \mathrm{a}-}$ $3.38 \mathrm{~d}$, and are very like those in QKI. They account for Liouvillian flow and collisions within the non-condensate-band.

3. Mean-field coupling term: One part of the interaction leads to a Hamiltonian coupling between the two bands, and this is a residual part of the Hamiltonian, which can be written

$$
H_{\mathrm{mf}}=2 u \int d^{3} \mathbf{x}\left\{\left(\sum_{\mathbf{Q}} \psi_{\mathbf{Q}}^{\dagger}(\mathbf{x}) \psi_{\mathbf{Q}}(\mathbf{x})-\bar{n}(\mathbf{x})\right)\right.
$$

$$
\left.\times\left(\phi^{\dagger}(\mathbf{x}) \phi(\mathbf{x})-\bar{\rho}(\mathbf{x})\right)\right\}
$$

Since this is only a residual after subtracting the mean-field terms, it is expected to be of the same order of magnitude as the irreversible terms. It is neglected entirely in writing the stationary density operator in the form 1.20.

4. Irreversible coupling terms: These are the irreversible terms which give rise to energy and particle transfer between the condensate-band and the non-condensate-band. They are written in terms of some formal condensate-band operators, and the field operators $\psi_{\mathbf{Q}}(\mathbf{x})$ of the non-condensate-band. In order to use the master equation, the eigenfunctions of $H_{\mathrm{C}}^{\mathrm{eff}}$, the effective condensate-band Hamiltonian, must be found. This is a question of choosing the appropriate approximation method.

\section{Practical methods}

By assuming that the non-condensate band can be maintained in a thermal stationary state we recover the methodology of QKIII. The next obvious step is to take a timedependent local equilibrium approximation for the noncondensate-band, and a number-conserving Bogoliubov approximation for the condensate-band. We can then talk about the system as a thermalized vapor in the non-condensate-band, in interaction with a system composed of a condensate and quasiparticles in the condensate-band. This is the most useful way of expressing our results. We find that

1. The condensate-band is described as the Bogoliubov system of quasiparticles and condensate, in which the mean-field potential induced by the vapor may be time-dependent.

2. The vapor is described by a quantum Boltzmann equation, in which the mean field potential induced by the condensate band may be time-dependent.

3. There are transfer terms between the condensate-band and vapor, which may be evaluated in various degrees of approximation.

\section{Non-condensate-band equations of motion}

The equation of motion for the phase-space density $f_{\mathbf{K}}(\mathbf{x})$ of the non-condensate-band vapor can be written

$$
\begin{aligned}
\frac{\partial f_{\mathbf{K}}(\mathbf{x})}{\partial t}= & \left(\frac{\hbar \mathbf{K} \cdot \nabla_{\mathbf{x}}}{m}-\frac{\nabla_{\mathbf{x}} V_{\mathrm{NC}}^{\mathrm{eff}}(\mathbf{x}, t) \cdot \nabla_{\mathbf{K}}}{\hbar}\right) f_{\mathbf{K}}(\mathbf{x}) \\
& +\frac{2|u|^{2}}{h^{2}} \int_{R_{\mathrm{NC}}} d^{3} \mathbf{K}_{2} \int_{R_{\mathrm{NC}}} d^{3} \mathbf{K}_{3} \int_{R_{\mathrm{NC}}} d^{3} \mathbf{K}_{4} \delta\left(\mathbf{K}+\mathbf{K}_{2}-\mathbf{K}_{3}-\mathbf{K}_{4}\right) \delta\left(\omega+\omega_{2}-\omega_{3}-\omega_{4}\right) \\
& \times\left\{f_{\mathbf{K}}(\mathbf{x}) f_{\mathbf{K}_{2}}(\mathbf{x})\left[f_{\mathbf{K}_{3}}(\mathbf{x})+1\right]\left[f_{\mathbf{K}_{4}}(\mathbf{x})+1\right]-\left[f_{\mathbf{K}}(\mathbf{x})+1\right]\left[f_{\mathbf{K}_{2}}(\mathbf{x})+1\right] f_{\mathbf{K}_{3}}(\mathbf{x}) f_{\mathbf{K}_{4}}(\mathbf{x})\right\}
\end{aligned}
$$




$$
+\left.\frac{\partial f_{\mathbf{K}}(\mathbf{x})}{\partial t}\right|_{1}+\left.\frac{\partial f_{\mathbf{K}}(\mathbf{x})}{\partial t}\right|_{2}+\left.\frac{\partial f_{\mathbf{K}}(\mathbf{x})}{\partial t}\right|_{3}
$$

The final three terms represent transfers of energy and atoms to and from the condensate band in which respectively 1,2 and 3 condensate-band field operators are involved in the matrix elements.

The transfer terms are given in full in 6.12), 6.22) and 6.25), and are necessarily rather complicated because of the mixing of creation and destruction operators generated by the Bogoliubov method. However, the essence of the result is that there are simple rate processes involving non-condensateband particle numbers, condensate band quasiparticle numbers, and the number of particles in the condensate. All of the rates involve integrals like

$$
\begin{aligned}
& \int d^{3} \mathbf{K}_{2} \int d^{3} \mathbf{K}_{3} \int d^{3} \mathbf{k} \delta\left(\omega_{2 \mathbf{K} 4}(\mathbf{x})-\omega\right) \delta\left(\mathbf{K}+\mathbf{K}_{4}-\mathbf{K}_{2}-\mathbf{k}\right) \\
& \times\left[1+f_{\mathbf{K}}(\mathbf{x})\right]\left[1+f_{\mathbf{K}_{4}}(\mathbf{x})\right] f_{\mathbf{K}_{2}}(\mathbf{x}) \mathcal{W}_{I}(\mathbf{x}, \mathbf{k}) .
\end{aligned}
$$

The quantity $\mathcal{W}_{I}(\mathbf{x}, \mathbf{k})$ is a Wigner function of a quasiparticle amplitude-we see therefore that there are energy and momentum conservation delta functions as in the quantum Boltzmann equation, and where a particle is in the condensate band we replace its phase space density by the Wigner function corresponding to the particular quasiparticle amplitude for the process being considered. Thes amplitudes are closely related to the functions $p_{m}(\mathbf{x})$, and $q_{m}(\mathbf{x})$ in equation (1.19). The effective potential $V^{\text {eff }}(\mathrm{x})$ experienced by the vapor as a result of the existence of a condensate and its quasiparticles.

$$
\begin{aligned}
V^{\mathrm{eff}}(\mathbf{x})= & 2 u \bar{\rho}(\mathbf{x}) \\
= & 2 u N\left|\xi_{N}(\mathbf{x})\right|^{2} \\
& +\sum_{m}\left\{\bar{n}_{m}\left|p_{m}(\mathbf{x})\right|^{2}+\left(\bar{n}_{m}+1\right)\left|q_{m}(\mathbf{x})\right|^{2}\right\}
\end{aligned}
$$

where the mean occupation number of the quasiparticle $m$ is defined in terms of the operators of (1.19) and the condensate band density operator $\rho_{\mathrm{C}}$ :

$$
\bar{n}_{m}=\operatorname{Tr}\left(b_{m}^{\dagger} b_{m} \rho_{\mathrm{C}}\right),
$$

and is time-dependent if $\rho_{\mathrm{C}}$ is time-dependent.

\section{Condensate-band equations of motion}

The methodology used does not demand that any further approximation be made to the condensate-band effective Hamiltonian as given in (1.13). However the interactions with the non-condensate band are most naturally expressed in terms of quasiparticles, as explained in Sect.[B3, and this will only make sense when the condensate-band Hamiltonian is expressed in terms of the Bogoliubov theory. To a first approximation it is reasonable to treat the condensate-band as a condensate plus a gas of non-interacting quasiparticles, and this is all we do explicitly in this paper.
There is no difficulty in principle in including the interactions between the quasiparticles which would arise in a full expansion of the condensate-band Hamiltonian using the quasiparticle states as a basis. However a practical method of doing this would probably depend on the specific problem under consideration.

\section{USE OF WAVELETS FOR FOR TRAPPED ATOMS}

In QKI we introduced a wavelet expansion of the field operators. The wavelets are a complete set of orthonormal oneparticle wavefunctions which are only significantly different from zero in a phase space volume $h^{3}$ around a point $\hbar \mathbf{K}, \mathbf{r}$ in phase space. By this we mean that the wavelet function $v_{\mathbf{K}}(\mathbf{x}, \mathbf{r})$ is only significantly different from zero in a coordinate space volume $\pi^{3} / \Delta^{3}$ around $\mathbf{r}$, and that their expression in terms of momentum eigenfunctions with eigenvalue $\mathbf{p}$ is only significantly different from zero in a momentum space volume $(2 \hbar \Delta)^{3}$ around $\mathbf{p}=\hbar \mathbf{K}$. This expansion enables one to express the quantum states of the Bose gas in terms of the numbers of particles associated with the wavelet $\mathbf{K}, \mathbf{r}$; which we can loosely think of as the number of particles in a phase space "cell" of volume $h^{3}$ at the point $\mathbf{K}, \mathbf{r}$. This gives a fully quantum mechanical description of the physics, but also connects very directly the classical description of the particle as having position $\mathbf{r}$, and momentum $\hbar \mathbf{K}$. Using the description, we can talk about scattering between different momentum values (the Boltzmann collision term), and flow through phase space (the Boltzmann streaming term). Because it is fully quantum mechanical, it is reasonably straightforward to use this phase space description for the non-condensate band $R_{\mathrm{NC}}$ at the same time as we use a description in terms of energy eigenfunctions in the condensate band $R_{\mathrm{C}}$.

\section{A. Formulation of wavelets}

\section{Wavelet properties}

The details of the construction of the wavelets are contained in Appendix A. The essential properties of the wavelets which we shall use here are as follows. We write the one-particle trapping Hamiltonian as

$$
H_{T}^{\text {(one particle) }}=\frac{\mathbf{p}^{2}}{2 m}+V(\mathbf{x})
$$

we can choose a set of wavelet functions $w_{\mathbf{Q}}(\mathbf{x}, \mathbf{r})$ with the following properties.

1. The wavelets are a complete orthonormal set 


$$
\begin{aligned}
\int d^{3} \mathbf{x} w_{\mathbf{Q}}^{*}(\mathbf{x}, \mathbf{r}) w_{\mathbf{Q}^{\prime}}\left(\mathbf{x}, \mathbf{r}^{\prime}\right) & =\delta_{\mathbf{Q}_{\mathbf{Q}^{\prime}}} \delta_{\mathbf{r r}^{\prime}} \\
\sum_{\mathbf{Q}, \mathbf{r}} w_{\mathbf{Q}}^{*}(\mathbf{x}, \mathbf{r}) w_{\mathbf{Q}}\left(\mathbf{x}^{\prime}, \mathbf{r}\right) & =\delta\left(\mathbf{x}-\mathbf{x}^{\prime}\right)
\end{aligned}
$$

2. The wavelet function $w_{\mathbf{Q}}(\mathbf{x}, \mathbf{r})$ is only significantly different from zero when $\mathrm{x} \approx \mathrm{r}$.

3. The mean energy of the wavelet is independent of $\mathbf{r}$, and is

$$
E(\mathbf{Q})=\frac{\hbar^{2} \mathbf{Q}^{2}}{2 m}
$$

The mean momentum associated with a wavelet is a vector $\hbar \mathbf{K}(\mathbf{Q}, \mathbf{r})$ which is such that

$$
\frac{\hbar^{2} \mathbf{Q}^{2}}{2 m}=\frac{\hbar^{2}(\mathbf{K}(\mathbf{Q}, \mathbf{r}))^{2}}{2 m}+V(\mathbf{r})
$$

The direction of $\mathbf{Q}$ is determined by the condition that the $\mathrm{Li}$ ouvillian flow in phase space does not change $Q$; this means that $\mathbf{K}(\mathbf{Q}, \mathbf{r})$ can be inverted to give a function $\mathbf{Q}(\mathbf{K}, \mathbf{r})$, which can be regarded as a smooth function of continuous variable on a sufficiently large scale, and that this function satisfies the equation

$$
\left\{\frac{\hbar \mathbf{K} \cdot \nabla_{\mathbf{r}}}{m}-\frac{\nabla V(\mathbf{r}) \cdot \nabla_{\mathbf{K}}}{\hbar}\right\} \mathbf{Q}(\mathbf{K}, \mathbf{r})=0 .
$$

Clearly, this is compatible with 2.5. We can fix the function by requiring that

$$
V(\mathbf{r})=0 \quad \Longrightarrow \quad \mathbf{Q}(\mathbf{K}, \mathbf{r})=\mathbf{K} .
$$

These requirements can be all satisfied simultaneously, as is demonstrated in Appendix A.

\section{Field operator in terms of wavelets}

1. The field operator can be expressed as

$$
\begin{aligned}
\psi(\mathbf{x}) & =\sum_{\mathbf{Q}, \mathbf{r}} w_{\mathbf{Q}}(\mathbf{x}, \mathbf{r}) A_{\mathbf{Q}, \mathbf{r}} \\
& =\sum_{\mathbf{Q}} \psi_{\mathbf{Q}}(\mathbf{x})
\end{aligned}
$$

and

$$
\left[A_{\mathbf{Q}, \mathbf{r}}, A_{\mathbf{Q}^{\prime}, \mathbf{r}^{\prime}}^{\dagger}\right]=\delta_{\mathbf{Q Q}^{\prime}} \delta_{\mathbf{r r}^{\prime}}
$$

2. For $\mathbf{r}, \mathbf{Q}$ sufficiently large that they may be regarded as being almost continuous variables, the commutator of the $\psi_{\mathbf{Q}}(\mathbf{x})$ functions has the asymptotic form

$$
\begin{gathered}
{\left[\psi_{\mathbf{Q}}(\mathbf{x}), \psi_{\mathbf{Q}^{\prime}}^{\dagger}\left(\mathbf{x}^{\prime}\right)\right]=\delta_{\mathbf{Q}, \mathbf{Q}^{\prime}} \sum_{\mathbf{r}} w_{\mathbf{Q}, \mathbf{r}}(\mathbf{x}) w_{\mathbf{Q}, \mathbf{r}}^{*}\left(\mathbf{x}^{\prime}\right)} \\
\approx \delta_{\mathbf{Q}, \mathbf{Q}^{\prime}} e^{i \mathbf{K}\left(\mathbf{Q}, \frac{\mathbf{x}+\mathbf{x}^{\prime}}{2}\right) \cdot\left(\mathbf{x}-\mathbf{x}^{\prime}\right)} g_{\mathbf{Q}}\left(\mathbf{x}, \mathbf{x}^{\prime}\right) .
\end{gathered}
$$

where the function $g_{\mathbf{Q}}\left(\mathbf{x}, \mathbf{x}^{\prime}\right)$ is only significantly different from zero when $\mathrm{x} \approx \mathrm{x}^{\prime}$, and is normalized to

$$
\int d^{3} \mathbf{x}^{\prime} g_{\mathbf{Q}}\left(\mathbf{x}, \mathbf{x}^{\prime}\right)=\int d^{3} \mathbf{x} g_{\mathbf{Q}}\left(\mathbf{x}, \mathbf{x}^{\prime}\right)=1
$$

The region of validity of this asymptotic form coincides essentially with that of the WKB approximation for the wavefunctions.

\section{Expression of the many particle Hamiltonian}

1. The many particle trapping Hamiltonian-that is, excluding interactions (1.3) — can be expressed as

$$
\begin{aligned}
H_{T}+H_{\mathrm{kin}}= & \sum_{\mathbf{r}} \sum_{\mathbf{Q}} E(\mathbf{Q}) A_{\mathbf{Q}, \mathbf{r}}^{\dagger} A_{\mathbf{Q}, \mathbf{r}} \\
& +\sum_{\mathbf{r} \mathbf{r}^{\prime}} \sum_{\mathbf{Q}} \mathcal{M}\left(\mathbf{Q}, \mathbf{r}, \mathbf{r}^{\prime}\right) A_{\mathbf{Q}, \mathbf{r}}^{\dagger} A_{\mathbf{Q}, \mathbf{r}^{\prime}}
\end{aligned}
$$

2. The object $\mathcal{M}\left(\mathbf{Q}, \mathbf{r}, \mathbf{r}^{\prime}\right)$ represents the quantized version of the Liouvillian motion, and defines a quantity $M\left(\mathbf{K}, \mathbf{K}^{\prime}, \mathbf{r}, \mathbf{r}^{\prime}\right)$ through

$$
M\left(\mathbf{K}(\mathbf{Q}, \mathbf{r}), \mathbf{K}\left(\mathbf{Q}, \mathbf{r}^{\prime}\right), \mathbf{r}, \mathbf{r}^{\prime}\right)=\mathcal{M}\left(\mathbf{Q}, \mathbf{r}, \mathbf{r}^{\prime}\right),
$$

a definition which is only valid for $(\mathbf{r}, \mathbf{K}),\left(\mathbf{r}^{\prime}, \mathbf{K}^{\prime}\right)$ on the same $\mathbf{Q}$ surface. When applied to a sufficiently smooth function $F(\mathbf{K}, \mathbf{r})$, the $M$ function generates the Liouvillian motion through

$$
\begin{aligned}
& \sum_{\mathbf{K}^{\prime}, \mathbf{r}^{\prime}} M\left(\mathbf{K}, \mathbf{K}^{\prime}, \mathbf{r}, \mathbf{r}^{\prime}\right) F\left(\mathbf{K}^{\prime}, \mathbf{r}^{\prime}\right) \\
& \quad \approx i \hbar\left\{\frac{\hbar \mathbf{K} \cdot \nabla_{\mathbf{r}}}{m}-\frac{\nabla V(\mathbf{r}) \cdot \nabla_{\mathbf{K}}}{\hbar}\right\} F(\mathbf{K}, \mathbf{r}) .
\end{aligned}
$$

3. The interaction part of the many-particle Hamiltonian, $H_{\mathrm{I}}$, (1.3) can be written in the form

$$
H_{\mathrm{I}}=\frac{1}{2} \sum_{1234} \mathcal{U}_{\mathbf{Q}_{1}, \mathbf{Q}_{2}, \mathbf{Q}_{3}, \mathbf{Q}_{4}}
$$

in which

$$
\begin{aligned}
& \mathcal{U}_{\mathbf{Q}_{1}, \mathbf{Q}_{2}, \mathbf{Q}_{3}, \mathbf{Q}_{4}} \\
& =\int d^{3} \mathbf{x} \int d^{3} \mathbf{x}^{\prime} u\left(\mathbf{x}-\mathbf{x}^{\prime}\right) \psi_{\mathbf{Q}_{1}}^{\dagger}(\mathbf{x}) \psi_{\mathbf{Q}_{2}}^{\dagger}\left(\mathbf{x}^{\prime}\right) \psi_{\mathbf{Q}_{3}}\left(\mathbf{x}^{\prime}\right) \psi_{\mathbf{Q}_{4}}(\mathbf{x})
\end{aligned}
$$




\section{B. Definition of the condensate band and non condensate bands}

The formulation in terms of wavelets so far has been appropriate for a situation in which no condensation occurs. We now consider the situation in which there is significant condensation. There are two aspects to be considered:

1. We must define the condensate-band and the noncondensate-band in terms of the $\mathrm{Q}$-band formulation. The condensate band, which we will call $R_{\mathrm{C}}$, will consist of all $\mathrm{Q}$ with energy less than a value $E_{\mathrm{R}}$, that is all $\mathrm{Q}$ such that $\hbar^{2} \mathbf{Q}^{2} / 2 m<E_{\mathrm{R}}$. The non-condensate band, which we will call $R_{\mathrm{NC}}$, consists of the complement of $R_{\mathrm{C}}$; that is, all states for which $\hbar^{2} \mathbf{Q}^{2} / 2 m \geq E_{\mathrm{R}}$. The non-condensate-band will be described in terms of wavelets, while the condensate-band will be described in terms of many-body eigenfunctions.

2. If there is significant condensation, there will be significant mean-field effects, which will give rise to a correction to the the trapping potential, as discussed in Sect.IB 2. For the noncondensate-band this means that the definition of the wavelets used will be changed, because these are defined in terms of a particular potential, as in (2.1).

\section{Commutation relations}

The procedure we will now follow is to resolve the field operators into Q-bands only within the non-condensate band, where we will find that the Q-bands are effectively thermalized, and are thus uncorrelated with each other. In the condensate band, significant correlation effects may arise because the interactions with the condensate are not negligible in this band-this leads to the use of a single field operator the describe the behavior of the whole of $R_{\mathrm{C}}$.

We now define the non-condensate band field operator $\psi_{\mathrm{NC}}(\mathbf{x})$, the field operator restricted to $R_{\mathrm{NC}}$, based on the Q-band form for the field operator (2.8,2.9), as

$$
\begin{aligned}
\psi_{\mathrm{NC}}(\mathbf{x}) & \equiv \sum_{\mathbf{Q}}^{\mathrm{NC}} \sum_{\mathbf{r}} w_{\mathbf{Q}}(\mathbf{x}, \mathbf{r}) A_{\mathbf{Q}, \mathbf{r}} \\
& \equiv \sum_{\mathbf{Q}}^{\mathrm{NC}} \psi_{\mathbf{Q}}(\mathbf{x})
\end{aligned}
$$

where the notation $\sum_{\mathrm{Q}}^{\mathrm{NC}}$ means the summation over $\mathbf{Q} \in$ $R_{\mathrm{NC}}$, with a corresponding notation for summations in $R_{\mathrm{C}}$.

The condensate band field operator will be called $\phi(\mathbf{x})$, and is defined by

$$
\phi(\mathbf{x}) \equiv \psi(\mathbf{x})-\psi_{\mathrm{NC}}(\mathbf{x})
$$

The commutation relations for these operators are

$$
\begin{aligned}
{\left[\phi(\mathbf{x}), \phi^{\dagger}\left(\mathbf{x}^{\prime}\right)\right] } & =\sum_{\mathbf{Q}}^{\mathrm{C}} \sum_{\mathbf{r}} w_{\mathbf{Q}, \mathbf{r}}(\mathbf{x}) w_{\mathbf{Q}, \mathbf{r}}^{*}\left(\mathbf{x}^{\prime}\right) \\
& \equiv g_{\mathrm{C}}\left(\mathbf{x}, \mathbf{x}^{\prime}\right)
\end{aligned}
$$

The $\phi$ and $\psi_{\mathbf{Q}}$ operators commute with each other, and the commutation relations of the $\psi_{\mathbf{Q}}(\mathbf{x})$ are as in 2.11,2.12)

\section{Separation of condensate and non-condensate parts of the full Hamiltonian}

We can now write the full Hamiltonian in a form which separates the three components; namely, those which act within $R_{\mathrm{NC}}$ only, those which act within $R_{\mathrm{C}}$ only, and those which cause transfers of energy or population between $R_{\mathrm{C}}$ and $R_{\mathrm{NC}}$. Thus we write the trapping plus kinetic parts of the Hamiltonian as the sum of three terms

$$
H_{\mathrm{kin}}+H_{T}=H_{a}+H_{b}+H_{\mathrm{C}, 1}
$$

in which the part which gives the average energy of the noncondensate atoms in each $\mathbf{Q}$-band is

$$
H_{a}=\sum_{\mathbf{r}} \sum_{\mathbf{Q}}^{\mathrm{NC}} \frac{\hbar^{2} \mathbf{Q}^{2}}{2 m} A_{\mathbf{Q}, \mathbf{r}}^{\dagger} A_{\mathbf{Q}, \mathbf{r}}
$$

The part which represents Liouvillian transport of the noncondensate atoms in each $\mathbf{Q}$-band is

$$
H_{b}=\sum_{\mathbf{r} \mathbf{r}^{\prime}} \sum_{\mathbf{Q}}^{\mathrm{NC}} \mathcal{M}\left(\mathbf{Q}, \mathbf{r}, \mathbf{r}^{\prime}\right) A_{\mathbf{Q}, \mathbf{r}}^{\dagger} A_{\mathbf{Q}, \mathbf{r}^{\prime}}
$$

The part which represents the non-interaction part of the condensate-band Hamiltonian is

$$
H_{\mathrm{C}, 1}=\int d^{3} \mathbf{x} \phi^{\dagger}(\mathbf{x})\left(-\frac{\hbar^{2} \nabla^{2}}{2 m}+V_{T}(\mathbf{x})\right) \phi(\mathbf{x}) .
$$

The interaction part $H_{\mathrm{I}}$ as defined in (1.3) can now be resolved into:

1. The part involving only $\phi$ operators, which is the self interaction within $R_{\mathrm{C}}$, which we call

$$
H_{\mathrm{C}, 2} \equiv \int d^{3} \mathbf{x} \int d^{3} \mathbf{x}^{\prime} \frac{u\left(\mathbf{x}-\mathbf{x}^{\prime}\right)}{2} \phi^{\dagger}(\mathbf{x}) \phi^{\dagger}\left(\mathbf{x}^{\prime}\right) \phi(\mathbf{x}) \phi\left(\mathbf{x}^{\prime}\right)
$$

2. The part involving no $\phi$ operators, which gives rise to scattering of the particles within $R_{\mathrm{NC}}$, which we call

$$
\begin{aligned}
& H_{\mathrm{I}, \mathrm{NC}} \equiv \\
& \int d^{3} \mathbf{x} \int d^{3} \mathbf{x}^{\prime} \frac{u\left(\mathbf{x}-\mathbf{x}^{\prime}\right)}{2} \psi_{\mathrm{NC}}^{\dagger}(\mathbf{x}) \psi_{\mathrm{NC}}^{\dagger}\left(\mathbf{x}^{\prime}\right) \psi_{\mathrm{NC}}(\mathbf{x}) \psi_{\mathrm{NC}}\left(\mathbf{x}^{\prime}\right) .
\end{aligned}
$$

3. There are finally the terms involving operators from both bands, which cause transfer of energy and/or particles between $R_{\mathrm{C}}$ and $R_{\mathrm{NC}}$. We call the parts involving one $\phi$ operator 


$$
\begin{aligned}
& H_{\mathrm{I}, \mathrm{C}}^{(1)} \equiv \\
& \int d^{3} \mathbf{x} \int d^{3} \mathbf{x}^{\prime} u\left(\mathbf{x}-\mathbf{x}^{\prime}\right) \psi_{\mathrm{NC}}^{\dagger}(\mathbf{x}) \psi_{\mathrm{NC}}^{\dagger}\left(\mathbf{x}^{\prime}\right) \psi_{\mathrm{NC}}(\mathbf{x}) \phi\left(\mathbf{x}^{\prime}\right) \\
& +\int d^{3} \mathbf{x} \int d^{3} \mathbf{x}^{\prime} u\left(\mathbf{x}-\mathbf{x}^{\prime}\right) \phi^{\dagger}(\mathbf{x}) \psi_{\mathrm{NC}}^{\dagger}\left(\mathbf{x}^{\prime}\right) \psi_{\mathrm{NC}}(\mathbf{x}) \psi_{\mathrm{NC}}\left(\mathbf{x}^{\prime}\right) .
\end{aligned}
$$

The parts involving two $\phi$ operators are called

$$
\begin{aligned}
& H_{\mathrm{I}, \mathrm{C}}^{(2)} \equiv \\
& \int d^{3} \mathbf{x} \int d^{3} \mathbf{x}^{\prime} u\left(\mathbf{x}-\mathbf{x}^{\prime}\right) \psi_{\mathrm{NC}}^{\dagger}(\mathbf{x}) \phi^{\dagger}\left(\mathbf{x}^{\prime}\right) \psi_{\mathrm{NC}}(\mathbf{x}) \phi\left(\mathbf{x}^{\prime}\right) \\
& +\int d^{3} \mathbf{x} \int d^{3} \mathbf{x}^{\prime} u\left(\mathbf{x}-\mathbf{x}^{\prime}\right) \phi^{\dagger}(\mathbf{x}) \psi_{\mathrm{NC}}^{\dagger}\left(\mathbf{x}^{\prime}\right) \psi_{\mathrm{NC}}(\mathbf{x}) \phi\left(\mathbf{x}^{\prime}\right) \\
& +\int d^{3} \mathbf{x} \int d^{3} \mathbf{x}^{\prime} \frac{u\left(\mathbf{x}-\mathbf{x}^{\prime}\right)}{2} \psi_{\mathrm{NC}}^{\dagger}(\mathbf{x}) \psi_{\mathrm{NC}}^{\dagger}\left(\mathbf{x}^{\prime}\right) \phi(\mathbf{x}) \phi\left(\mathbf{x}^{\prime}\right) \\
& +\int d^{3} \mathbf{x} \int d^{3} \mathbf{x}^{\prime} \frac{u\left(\mathbf{x}-\mathbf{x}^{\prime}\right)}{2} \phi^{\dagger}(\mathbf{x}) \phi^{\dagger}\left(\mathbf{x}^{\prime}\right) \psi_{\mathrm{NC}}(\mathbf{x}) \psi_{\mathrm{NC}}\left(\mathbf{x}^{\prime}\right)
\end{aligned}
$$

The parts involving three $\phi$ operators are called

$$
\begin{aligned}
& H_{\mathrm{I}, \mathrm{C}}^{(3)} \equiv \\
& \int d^{3} \mathbf{x} \int d^{3} \mathbf{x}^{\prime} u\left(\mathbf{x}-\mathbf{x}^{\prime}\right) \phi^{\dagger}(\mathbf{x}) \phi^{\dagger}\left(\mathbf{x}^{\prime}\right) \phi(\mathbf{x}) \psi_{\mathrm{NC}}\left(\mathbf{x}^{\prime}\right) \\
& +\int d^{3} \mathbf{x} \int d^{3} \mathbf{x}^{\prime} u\left(\mathbf{x}-\mathbf{x}^{\prime}\right) \psi_{\mathrm{NC}}^{\dagger}(\mathbf{x}) \phi^{\dagger}\left(\mathbf{x}^{\prime}\right) \phi(\mathbf{x}) \phi\left(\mathbf{x}^{\prime}\right) .
\end{aligned}
$$

Our notation is not entirely systematic, since $H_{\mathrm{I}, \mathrm{NC}}$ involves only the non-condensate-band operators, whereas $H_{\mathrm{I}, \mathrm{C}}$ involves operators from both the condensate-band and the noncondensate-band.

\section{Mean field corrections}

In practice the thermalized part of the condensate-vapor system consists of nearly all of the levels above the condensate level, and it is advantageous to include this fact explicitly as early as possible in the analysis. Thus, we would like to put the division between the condensate and non-condensate bands at as low an energy as possible. The main difference between the condensate band and the non-condensate band is the nature of the excitation spectrum. It is relatively simple to include corrections to the energy levels of the non-condensate band provided these energy levels are particle-like-that is, they are states with a definite energy and a definite number of particles. In fact, computations have shown only a small proportion of the excitation spectrum is not-particle like, and this is at quite low energy [25]. However, the energies of lower energy particle-like excitations are affected by the presence of the condensate and of each other. We therefore must consider mean field effects. There are three kinds of mean field effect which can be explicitly included:
1. The average effect of the condensate band on the noncondensate band;

2. The average effect of the non-condensate band on itself;

3 . The average effect of the the non-condensate band on the condensate band.

Our aim will be to estimate these average effects explicitly, and include them in the two Hamiltonians used to describe the two bands. The sum of these two terms is then subtracted from the interaction part of the Hamiltonian, which is preserved as a correction term. The description is thus in principle exact, since the total Hamiltonian is not changed-only the basis for the perturbation theory is changed.

If we suppose that $\bar{\rho}(\mathbf{x})$ is a c-number estimate of the condensate band density, and $\bar{n}(\mathbf{x})$ is a c-number estimate of the non-condensate band density, then the mean field effects 1 . and 2. give an equivalent potential acting on the noncondensate

$$
V_{\mathrm{NC}}^{\mathrm{mf}}(\mathbf{x})=2 u(\bar{\rho}(\mathbf{x})+\bar{n}(\mathbf{x}))
$$

while the effect 3 . is given by an equivalent potential acting on the condensate

$$
V_{\mathrm{C}}^{\mathrm{mf}}(\mathbf{x})=2 u \bar{n}(\mathbf{x})
$$

The mean field effect is included by adding and subtracting a term

$$
\int d^{3} \mathbf{x}\left\{V_{\mathrm{NC}}^{\mathrm{mf}}(\mathbf{x}) \psi_{\mathrm{NC}}^{\dagger}(\mathbf{x}) \psi_{\mathrm{NC}}(\mathbf{x})+V_{\mathrm{C}}^{\mathrm{mf}}(\mathbf{x}) \phi^{\dagger}(\mathbf{x}) \phi(\mathbf{x})\right\} .
$$

Effectively, this changes the potentials used in defining the two bands. For the non-condensate band, the change is

$$
V_{T}(\mathbf{x}) \rightarrow V_{\mathrm{NC}}^{\mathrm{eff}}(\mathbf{x})=V_{T}(\mathbf{x})+V_{\mathrm{NC}}^{\mathrm{mf}}(\mathbf{x}),
$$

with a consequent redefinition of wavelets. Thus the non-condensate band Hamiltonian retains the form as in 2.24,2.25, but the wavelets are now defined in terms of the effective potential $V_{\mathrm{NC}}^{\mathrm{eff}}(\mathbf{x})$.

For the condensate the change is

$$
V_{T}(\mathbf{x}) \rightarrow V_{\mathrm{C}}^{\mathrm{eff}}(\mathbf{x})=V_{T}(\mathbf{x})+V_{\mathrm{C}}^{\mathrm{mf}}(\mathbf{x}) .
$$

which now transforms the $H_{\mathrm{C}}$ to $H_{\mathrm{C}}^{\text {eff }}$, defined by

$$
H_{\mathrm{C}}^{\mathrm{eff}}=\int d^{3} \mathbf{x} \phi^{\dagger}(\mathbf{x})\left(-\frac{\hbar^{2} \nabla^{2}}{2 m}+V_{\mathrm{C}}^{\mathrm{eff}}(\mathbf{x})\right) \phi(\mathbf{x})+H_{\mathrm{C}, 2} .
$$

The extra terms are then subtracted off the interaction part of the Hamiltonian, giving

$$
\begin{aligned}
H_{\mathrm{I}, \mathrm{C}}^{(2)} \rightarrow & H_{\mathrm{I}, \mathrm{C}}^{(2, \text { eff })} \\
= & H_{\mathrm{I}, \mathrm{C}}^{(2)}-\int d^{3} \mathbf{x} V_{\mathrm{NC}}^{\mathrm{mf}}(\mathbf{x}) \psi_{\mathrm{NC}}^{\dagger}(\mathbf{x}) \psi_{\mathrm{NC}}(\mathbf{x}) \\
& -\int d^{3} \mathbf{x} V_{\mathrm{C}}^{\mathrm{mf}}(\mathbf{x}) \phi(\mathbf{x}) \phi^{\dagger}(\mathbf{x}) .
\end{aligned}
$$


We use the term $H_{\mathrm{I}, \mathrm{C}}^{\mathrm{eff}}$ for the sum of all of these interaction terms;

$$
H_{\mathrm{I}, \mathrm{C}}^{\mathrm{eff}} \equiv H_{\mathrm{I}, \mathrm{C}}^{(1)}+H_{\mathrm{I}, \mathrm{C}}^{(2, \mathrm{eff})}+H_{\mathrm{I}, \mathrm{C}}^{(3)} .
$$

The description is still exact, since the mean-field terms have been added and subtracted, but an estimate of the mean effect of the interaction has been explicitly included in the basis description of the condensate and non-condensate. The methodology does not depend on the particular choices of (2.32.2.33). It is possible, for example

1. To choose the mean field potentials to be fixed quantities;

2. To choose them to depend on the numbers of particles in the condensate and non-condensate bands;

3. To include an explicit time dependence. However, if this is done, the derivation and form of the master equation in Sect. [II] would be altered.

The choice of the estimates for $\bar{n}(\mathbf{x})$ and $\bar{\rho}(\mathbf{x})$ is not made at this stage, but will be done as part of the derivation of the master equation, since their choice must be such as to optimize the basis in which the master equation is described.

\section{DERIVATION OF THE MASTER EQUATION}

Using the separation into condensate band and noncondensate band operators we can now proceed to develop a master equation in a a way that combines the methodologies of QKI and QKIII.

\section{A. Projectors in the Q-bands}

We can now introduce the definitions of the projectors which we shall need, in much the same way as in QKI. The states of the system will be written in the form

$$
\left|\Psi_{\mathrm{C}}, \mathbf{n}\right\rangle
$$

in which $\Psi_{\mathrm{C}}$ represents the condensate degrees of freedom, and $\mathbf{n}$ is a vector of integers $n_{\mathbf{Q}, \mathbf{r}}$ representing the numbers of atoms in the $\mathbf{Q}$-cell $\mathbf{Q}, \mathbf{r}$. We will want to consider a projector definition of the form

$$
\begin{aligned}
p_{\mathbf{N}}\left|\Psi_{\mathrm{C}}, \mathbf{n}\right\rangle & =\left|\Psi_{\mathrm{C}}, \mathbf{n}\right\rangle & & \text { if } \sum_{\mathbf{r}} \mathbf{n}\left(\mathbf{Q}^{\prime}, \mathbf{r}\right)=N(\mathbf{Q}), \\
& =0 & & \text { otherwise. }
\end{aligned}
$$

This projector projects only in $R_{\mathrm{NC}}$, leaving the condensate state description $\Psi_{\mathrm{C}}$ completely unaffected.

The projectors on the density operator are defined as in QKI

$$
\begin{aligned}
\mathcal{P}_{\mathbf{N}} \rho & \equiv p_{\mathbf{N}} \rho p_{\mathbf{N}} \\
& \equiv v_{\mathbf{N}} .
\end{aligned}
$$

The complementary projector $\mathcal{Q}$ is defined by

$$
\mathcal{Q}=1-\sum_{\mathbf{N}} \mathcal{P}_{\mathbf{N}}
$$

where, by the definition (3.2) of $\mathcal{P}_{\mathbf{N}}$, the range of the index $\mathbf{N}$ includes configurations entirely within $R_{\mathrm{NC}}$.

Thus the terms $v_{\mathbf{N}}$ will be the parts of the density operator for which we can derive a master equation, while the complementary part

$$
w \equiv \mathcal{Q} \rho
$$

is the part which is neglected.

The quantities $v_{\mathbf{N}}$ are thus density operators in which $\mathbf{N}$ represents the $\mathbf{Q}$-band configuration of non-condensate-band particles, and in which no restriction of any kind is placed on the configuration of the particles in the condensate band.

\section{B. Formal derivation of the master equation}

The formal derivation of the master equation follows much the same methodology as in QKI and QKIII. In the present situation, for the purposes of developing the master equation we can divide the operators into the various parts as in Sec.IIB 2, but also taking account of the fact that we use the effective Hamiltonians which include estimates of the mean field effects:

$$
\begin{array}{cl}
H_{\mathrm{C}}^{\mathrm{eff}}=H_{\mathrm{C}, 1}^{\mathrm{eff}}+H_{\mathrm{C}, 2} & {[\text { see }(2.37)],} \\
H_{\mathrm{I}}^{\mathrm{eff}}=H_{\mathrm{I}, \mathrm{NC}}^{\mathrm{eff}}+H_{\mathrm{I}, \mathrm{C}}^{\mathrm{eff}} & {[\text { see } 2.38,2.39],} \\
H_{\mathrm{NC}}^{\mathrm{eff}}=H_{a}^{\mathrm{eff}}+H_{b}^{\mathrm{eff}} . &
\end{array}
$$

In the last of these, we understand $H_{a}^{\text {eff }}$ and $H_{b}^{\text {eff }}$ to be constructed in the same way as $(2.24,2.25)$, but using the effective potential $V_{\mathrm{NC}}^{\text {eff }}(\mathbf{x})$ innstead of $V(\mathbf{x})$ in 2.1) as a basis for counstructing the wavelets.

There are the relations

$$
\begin{aligned}
{\left[H_{a}^{\text {eff }}, \mathcal{P}_{\mathbf{N}} \rho\right] } & =0 \\
\mathcal{P}_{\mathbf{N}} H_{b}^{\text {eff }} \rho & =H_{b}^{\text {eff }} \mathcal{P}_{\mathbf{N}} \rho \\
\mathcal{P}_{\mathbf{N}} H_{\mathrm{C}}^{\text {eff }} \rho & =H_{\mathrm{C}}^{\text {eff }} \mathcal{P}_{\mathbf{N}} \rho
\end{aligned}
$$

which follow directly from the construction of the operators and projectors.

The equation of motion is

$$
\begin{aligned}
\dot{\rho} & =-\frac{i}{\hbar}\left[H_{a}^{\mathrm{eff}}+H_{b}^{\mathrm{eff}}+H_{I}^{\mathrm{eff}}+H_{\mathrm{C}}^{\mathrm{eff}}, \rho\right] \\
& \equiv\left(\mathcal{L}_{a}+\mathcal{L}_{b}+\mathcal{L}_{I}+\mathcal{L}_{\mathrm{C}}\right) \rho .
\end{aligned}
$$

Notice that, for compactness, we do not explicitly write $\mathcal{L}^{\text {eff }}$ for the Liouvillians.

From this form it is straightforward to write the master equation in the same form as in QKI. As in QKI, we use the Laplace transform notation for any function $f(t)$

$$
\tilde{f}(s)=\int_{0}^{\infty} e^{-s t} f(t) d t .
$$


Using this notation and the relations 3.8 3.8 , the master equation takes the form

$$
\begin{aligned}
& s \tilde{v}_{\mathbf{N}}(s)-v_{\mathbf{N}}(0)=\mathcal{L}_{b} \tilde{v}_{\mathbf{N}}(s)+\mathcal{P}_{\mathbf{N}} \mathcal{L}_{I} \sum_{\mathbf{M}} \tilde{v}_{\mathbf{M}}(s) \\
& \quad+\mathcal{P}_{\mathbf{N}} \mathcal{L}_{I}\left[s-\mathcal{L}_{a}-\mathcal{L}_{b}-\mathcal{L}_{\mathrm{C}}-\mathcal{Q} \mathcal{L}_{I}\right]^{-1} \mathcal{Q} \mathcal{L}_{I} \sum_{\mathbf{M}} \tilde{v}_{\mathbf{M}}(s)
\end{aligned}
$$

In this form the master equation is basically exact. We shall make the approximation that the kernel of the second part, the []$^{-1}$ term, can be approximated by keeping only the terms which describe the effective Hamiltonians within $R_{\mathrm{C}}$ or $R_{\mathrm{NC}}$, namely the terms $\mathcal{L}_{a}$ and $\mathcal{L}_{\mathrm{C}}$. Thus the master equation we shall use is written

$$
\begin{aligned}
& s \tilde{v}_{\mathbf{N}}(s)-v_{\mathbf{N}}(0)=\mathcal{L}_{b} \tilde{v}_{\mathbf{N}}(s)+\mathcal{P}_{\mathbf{N}} \mathcal{L}_{I} \sum_{\mathbf{M}} \tilde{v}_{\mathbf{M}}(s) \\
& +\mathcal{P}_{\mathbf{N}} \mathcal{L}_{I}\left[s-\mathcal{L}_{a}-\mathcal{L}_{\mathrm{C}}\right]^{-1} \mathcal{Q} \mathcal{L}_{I} \sum_{\mathbf{M}} \tilde{v}_{\mathbf{M}}(s)
\end{aligned}
$$

This is essentially the same approximation as in QKI, apart from the inclusion of the condensate band self-interaction term in $\mathcal{L}_{\mathrm{C}}$. However it should be noted that $R_{\mathrm{C}}$ is a rather wide band compared to the the $\mathbf{K}=0$ band of QKI, and contains significant dynamics, which we do not assume to be thermalized-rather, we expect these levels to become thermalized as a consequence of the equations of motion.

\section{Reversible terms}

\section{Streaming Term}

This arises from the term $\mathcal{L}_{b} v_{\mathbf{N}}(t)$, and takes an essentially classical form, based on of (2.14 2.16), in the degree of approximation we shall later use.

\section{Mean-field and forward scattering terms}

These arise from the term $\mathcal{P}_{\mathbf{N}} \mathcal{L}_{I} \sum_{\mathbf{v}} \tilde{v}_{\mathbf{M}}(s)$. Using the quantity $\mathcal{U}_{\mathbf{Q}_{1}, \mathbf{Q}_{2}, \mathbf{Q}_{3}, \mathbf{Q}_{4}}$ as defined in (2.18), we find that these terms are equivalent to a Hamiltonian term of the form

$$
\begin{aligned}
& H_{\text {forward }} \equiv \frac{1}{2} \sum_{\mathbf{Q}_{1} \neq \mathbf{Q}_{2}}\left\{\mathcal{U}_{\mathbf{Q}_{1}, \mathbf{Q}_{2}, \mathbf{Q}_{1}, \mathbf{Q}_{2}}+\mathcal{U}_{\mathbf{Q}_{1}, \mathbf{Q}_{2}, \mathbf{Q}_{2}, \mathbf{Q}_{1}}\right\} \\
& +\sum_{\mathbf{Q}}\left\{\frac{1}{2} \mathcal{U}_{\mathbf{Q}, \mathbf{Q}, \mathbf{Q}, \mathbf{Q}}\right. \\
& +\int d^{3} \mathbf{x} \int d^{3} \mathbf{x}^{\prime} u\left(\mathbf{x}-\mathbf{x}^{\prime}\right) \psi_{\mathbf{Q}}^{\dagger}(\mathbf{x}) \phi^{\dagger}\left(\mathbf{x}^{\prime}\right) \phi\left(\mathbf{x}^{\prime}\right) \psi_{\mathbf{Q}}(\mathbf{x}) \\
& +\int d^{3} \mathbf{x} \int d^{3} \mathbf{x}^{\prime} u\left(\mathbf{x}-\mathbf{x}^{\prime}\right) \psi_{\mathbf{Q}}^{\dagger}(\mathbf{x}) \phi^{\dagger}\left(\mathbf{x}^{\prime}\right) \phi(\mathbf{x}) \psi_{\mathbf{Q}}\left(\mathbf{x}^{\prime}\right)
\end{aligned}
$$

$$
\begin{aligned}
& \left.-\int d^{3} \mathbf{x} V_{\mathrm{NC}}^{\operatorname{mf}}(\mathbf{x}) \psi_{\mathbf{Q}}^{\dagger}(\mathbf{x}) \psi_{\mathbf{Q}}(\mathbf{x})\right\} \\
& -\int d^{3} \mathbf{x} V_{\mathrm{C}}^{\mathrm{mf}}(\mathbf{x}) \phi^{\dagger}(\mathbf{x}) \phi(\mathbf{x}) .
\end{aligned}
$$

In (3.14) one can see clearly the "Hartree" term in the third line, and the "Fock" term in the fourth line. However in the remainder of this paper we shall use the delta function form of the interaction potential $u\left(\mathbf{x}-\mathbf{x}^{\prime}\right) \rightarrow u \delta\left(\mathbf{x}-\mathbf{x}^{\prime}\right)$, and the difference between these terms and the second and third lines of (2.30) disappears. None of our results will depend heavily on the validity of this approximation, but the formulae do become considerably more compact when it is made.

We thus arrive at the final form of the forward scattering term

$$
\begin{aligned}
& H_{\text {forward }} \rightarrow H_{\text {forward }}^{\text {eff }} \\
& =\sum_{\mathbf{Q}_{1} \neq \mathbf{Q}_{2}} \mathcal{U}_{\mathbf{Q}_{1}, \mathbf{Q}_{2}, \mathbf{Q}_{1}, \mathbf{Q}_{2}}+\sum_{\mathbf{Q}}\left\{\frac{1}{2} \mathcal{U}_{\mathbf{Q}, \mathbf{Q}, \mathbf{Q}, \mathbf{Q}}\right. \\
& \left.\quad+2 u \int d^{3} \mathbf{x} \psi_{\mathbf{Q}}^{\dagger}(\mathbf{x}) \psi_{\mathbf{Q}}(\mathbf{x})\left\{\phi^{\dagger}(\mathbf{x}) \phi(\mathbf{x})-\bar{\rho}(\mathbf{x})-\bar{n}(\mathbf{x})\right\}\right\} \\
& \quad-2 u \int d^{3} \mathbf{x} \bar{n}(\mathbf{x}) \phi^{\dagger}(\mathbf{x}) \phi(\mathbf{x})
\end{aligned}
$$

\section{Collisional terms}

The remaining parts of the master equation (3.12) lead to genuinely irreversible collision terms, both within the noncondensate band, and between the two bands.

\section{Collisions within the non-condensate band}

The interactions which involve only particles in $R_{\mathrm{NC}}$ can cause collisions between the particles in the noncondensate band. Thus we consider the parts of the second line of 3.13 which arise from the part

$$
\mathcal{P}_{\mathbf{N}} \mathcal{L}_{\mathrm{I}, \mathrm{NC}}\left[s-\mathcal{L}_{a}-\mathcal{L}_{\mathrm{C}}\right]^{-1} \mathcal{Q} \mathcal{L}_{\mathrm{I}, \mathrm{NC}} \sum_{\mathbf{M}} v_{\mathbf{M}}(s) .
$$

The result of this process can be seen by considering a particular term in the expansion of (3.16). The inversion of the Laplace transform would give a term like

$$
\mathcal{U}(1234) \int_{0}^{\infty} d \tau \exp \left\{\left(\mathcal{L}_{a}+\mathcal{L}_{\mathrm{C}}\right) \tau\right\} \mathcal{U}\left(4^{\prime} 3^{\prime} 2^{\prime} 1^{\prime}\right) v_{\mathbf{N}}(t-\tau)
$$

The only surviving term after projection will have

$$
\begin{aligned}
& 1=1^{\prime}, 2=2^{\prime} \text { or } 1=2^{\prime}, 2=1^{\prime} \\
& \text { and } \\
& 3=3^{\prime}, 4=4^{\prime} \text { or } 3=4^{\prime}, 4=3^{\prime}
\end{aligned}
$$


and we can write this as

$$
\begin{aligned}
& \mathcal{U}(1234) \int_{0}^{\infty} d \tau e^{-i \Delta \omega_{1234} \tau} \mathcal{U}(4321) \\
& \times e^{-\frac{i}{\hbar}\left(H_{a}+H_{\mathrm{C}}^{\text {eff }}\right) \tau} v_{\mathbf{N}}(t-\tau) e^{\frac{i}{\hbar}\left(H_{a}+H_{\mathrm{C}}^{\text {eff }}\right) \tau},
\end{aligned}
$$

where, as in QKI,

$$
\Delta \omega_{1234} \equiv \omega_{4}+\omega_{3}-\omega_{2}-\omega_{1}
$$

but where we use the notations

$$
\begin{aligned}
\hbar \omega_{\mathbf{Q}} & =\frac{\hbar^{2} \mathbf{Q}^{2}}{2 m}, \\
\omega_{i} & \equiv \omega_{\mathbf{Q}_{i}} .
\end{aligned}
$$

We now make the usual approximation that, in the time interval over which the $\tau$ integration is significant, we may ignore the irreversible part of the time evolution of $v_{\mathbf{N}}$, so that the exponential terms simply set $t-\tau \rightarrow t$, and we get simply

$$
\mathcal{U}(1234) \int_{0}^{\infty} d \tau e^{-i \Delta \omega_{1234} \tau} \mathcal{U}(4321) v_{\mathbf{N}}(t)
$$

which leads to the terms in the QKME which describe the scattering of the modes in $R_{\mathrm{NC}}$ in exactly the same form as in (QKI:75d,e).

\section{Interaction between $R_{\mathrm{C}}$ and $R_{\mathrm{NC}}$}

The treatment of these terms depends on writing the interaction Hamiltonian terms in a form which uses wavelet functions for the non-condensate-band operators, and energy eigenfunctions for the condensate band trap operators as follows:

$$
\begin{aligned}
H_{\mathrm{I}, \mathrm{C}}^{(1)} & =\sum_{234} \int d^{3} \mathbf{x} Z_{432}(\mathbf{x}) \sum_{m} X_{m}^{\dagger}(\mathbf{x})+\text { h.c. } \\
H_{\mathrm{I}, \mathrm{C}}^{(2, \mathrm{eff})} & \approx 2 \sum_{42} \int d^{3} \mathbf{x} Z_{42}(\mathbf{x}) \sum_{r} U_{r}^{\dagger}(\mathbf{x})+\text { h.c. } \\
H_{\mathrm{I}, \mathrm{C}}^{(3)} & =\sum_{4} \int d^{3} \mathbf{x} Z_{4}(\mathbf{x}) \sum_{r} V_{r}^{\dagger}(\mathbf{x})+\text { h.c. }
\end{aligned}
$$

The non-condensate band is represented by the $Z$ operators; an abbreviated notation for

$$
\begin{aligned}
Z_{432}(\mathbf{x}) & =u \psi_{4}(\mathbf{x}) \psi_{3}(\mathbf{x}) \psi_{2}^{\dagger}(\mathbf{x}) \\
Z_{42}(\mathbf{x}) & =u \psi_{4}(\mathbf{x}) \psi_{2}^{\dagger}(\mathbf{x}) \\
Z_{4}(\mathbf{x}) & =u \psi_{4}(\mathbf{x})
\end{aligned}
$$

The condensate band operators are defined by

$$
\begin{aligned}
\phi(\mathbf{x}) & =\sum_{m} X_{m}(\mathbf{x}) \\
\phi(\mathbf{x}) \phi^{\dagger}(\mathbf{x}) & =\sum_{r} U_{r}(\mathbf{x}), \\
\phi^{\dagger}(\mathbf{x}) \phi(\mathbf{x}) \phi(\mathbf{x}) & =\sum_{r} V_{r}(\mathbf{x}) .
\end{aligned}
$$

The $X, U, V$ operators are, as in QKIII (where they are discussed more extensively), eigenoperators of the condensateband effective Hamiltonian, with eigenvalues defined by

$$
\begin{array}{r}
{\left[H_{\mathrm{C}}^{\mathrm{eff}}, X_{m}(\mathbf{x})\right]=-\hbar \epsilon_{m} X_{m}(\mathbf{x}),} \\
{\left[H_{\mathrm{C}}^{\mathrm{eff}}, U_{r}(\mathbf{x})\right]=-\hbar \beta_{r} U_{r}(\mathbf{x}),} \\
{\left[H_{\mathrm{C}}^{\mathrm{eff}}, V_{r}(\mathbf{x})\right]=-\hbar \beta_{r}^{\prime} V_{r}(\mathbf{x}) .}
\end{array}
$$

In the Bogoliubov approximation, these operators have relatively simple expressions in terms of quasiparticle creation and destruction operators - the precise relationship is given in Sec.IID of QKIII; especially (94-99) which gives the basic definition, and Sec.IID3 which gives the expression of the more complicated $U_{r}(\mathbf{x})$ and $V_{r}(\mathbf{x})$ operators.

We omit terms involving $\phi(\mathbf{x}) \phi(\mathbf{x})$, or its Hermitian conjugate, since these are always very non-resonant. We use the rotaing wave, or random phase approximation as in QKIII, and derive a master equation which is a blend of the formalism of QKI and that of QKIII.

\section{E. Master equation terms}

Putting these all together we get the contribution to the master equation which describes coupling between $R_{\mathrm{C}}$ and $R_{\mathrm{NC}}$ so that the full quantum kinetic master equation now takes the form

$$
\dot{v}_{\mathbf{N}}(t)=\left.\dot{v}_{\mathbf{N}}(t)\right|_{\mathrm{C}}+\left.\dot{v}_{\mathbf{N}}(t)\right|_{\mathrm{NC}}+\left.\dot{v}_{\mathbf{N}}(t)\right|_{\mathrm{MF}}+\left.\dot{v}_{\mathbf{N}}(t)\right|_{\text {coupling }}
$$

in which the various terms are defined as follows.

\section{Condensate band term}

This is

$$
\left.\dot{v}_{\mathbf{N}}(t)\right|_{\mathbf{C}}=\left[H_{\mathrm{C}}^{\mathrm{eff}}, v_{\mathbf{N}}(t)\right]
$$

where, as in (2.37),

$$
H_{\mathrm{C}}^{\mathrm{eff}}=\int d^{3} \mathbf{x} \phi^{\dagger}(\mathbf{x})\left(-\frac{\hbar^{2} \nabla^{2}}{2 m}+V_{\mathrm{NC}}^{\mathrm{eff}}(\mathbf{x})\right) \phi(\mathbf{x})+\frac{u}{2} \int d^{3} \mathbf{x} \phi^{\dagger}(\mathbf{x}) \phi^{\dagger}(\mathbf{x}) \phi(\mathbf{x}) \phi(\mathbf{x})
$$




\section{Non-condensate band term}

$$
\begin{aligned}
\left.\dot{v}_{\mathbf{N}}(t)\right|_{\mathrm{NC}}= & -\frac{i}{\hbar}\left[H_{b}, v_{\mathbf{N}}(t)\right] \\
& -\frac{i}{\hbar}\left[H_{\mathrm{F}, \mathrm{NC}}, v_{\mathbf{N}}(t)\right] \\
& -\frac{i}{\hbar^{2}} \sum_{\mathbf{e}} \mathrm{P} \frac{1}{\Delta \omega(\mathbf{e})}\left[\mathcal{U}(\mathbf{e}) \mathcal{U}^{\dagger}(\mathbf{e}), v_{\mathbf{N}}(t)\right] \\
& +\frac{\pi}{\hbar^{2}} \sum_{\mathbf{e}} \delta(\Delta \omega(\mathbf{e}))\left\{2 \mathcal{U}(\mathbf{e}) v_{\mathbf{N}-\mathbf{e}}(t) \mathcal{U}^{\dagger}(\mathbf{e})-\mathcal{U}^{\dagger}(\mathbf{e}) \mathcal{U}(\mathbf{e}) v_{\mathbf{N}}(t)-v_{\mathbf{N}}(t) \mathcal{U}^{\dagger}(\mathbf{e}) \mathcal{U}(\mathbf{e})\right\}
\end{aligned}
$$

Here we define the quantity

$$
H_{\mathrm{F}, \mathrm{NC}}=\frac{1}{2} \sum_{\mathbf{Q}} \mathcal{U}_{\mathbf{Q}, \mathbf{Q}, \mathbf{Q}, \mathbf{Q}}+\sum_{\mathbf{Q}_{1} \neq \mathbf{Q}_{2}} \mathcal{U}_{\mathbf{Q}_{1}, \mathbf{Q}_{2}, \mathbf{Q}_{1}, \mathbf{Q}_{2}}-2 u \sum_{\mathbf{Q}} \psi_{\mathbf{Q}}^{\dagger}(\mathbf{x}) \psi_{\mathbf{Q}}(\mathbf{x}) \bar{n}(\mathbf{x}),
$$

which is that part of the forward scattering term (3.14) - including the mean field correction-and affects only the noncondensate band.

As in QKI we have defined the vector in the space of $\mathbf{M}, \mathbf{N}$, called $\mathbf{e}_{1234}$ which can be written

$$
\left.\mathbf{e}_{1234}\right|_{\mathbf{Q}_{1}}=\left.\mathbf{e}_{1234}\right|_{\mathbf{Q}_{2}}=-\left.\mathbf{e}_{1234}\right|_{\mathbf{Q}_{3}}=-\left.\mathbf{e}_{1234}\right|_{\mathbf{Q}_{4}}=1 .
$$

with all other components are zero. Thus $\mathbf{e}_{1234}$ represents a collision, and we have used the notation $\sum_{\mathbf{e}}$ as a shorthand for a summation over $\mathbf{Q}_{1}, \mathbf{Q}_{2}, \mathbf{Q}_{3}, \mathbf{Q}_{4}$.

\section{Mean-field coupling term}

This is the term which gives rise to mean-field effects, and arises from that part of the forward scattering term (3.14 $)$ which mixes together the condensate-band and non-condensate band operators. It is

$$
\left.\dot{v}_{\mathbf{N}}(t)\right|_{\mathrm{mf}}=-\frac{i}{\hbar}\left[H_{\mathrm{mf}}, v_{\mathbf{N}}(t)\right]
$$

with

$$
H_{\mathrm{mf}}=2 u \int d^{3} \mathbf{x}\left\{\left(\sum_{\mathbf{Q}} \psi_{\mathbf{Q}}^{\dagger}(\mathbf{x}) \psi_{\mathbf{Q}}(\mathbf{x})-\bar{n}(\mathbf{x})\right)\left(\phi^{\dagger}(\mathbf{x}) \phi(\mathbf{x})-\bar{\rho}(\mathbf{x})\right)\right\}
$$

This term is a relatively small coupling term, since the major mean field effects have been canceled. For ease of writing, a cnumber term $2 \int d^{3} \mathbf{x} \bar{n}(\mathbf{x}) \bar{\rho}(\mathbf{x})$ has been added, leading to the factorized form given. Of course this does not affect the equations of motion.

\section{Coupling terms}

These terms give all transfer of energy and population between $R_{\mathrm{C}}$ and $R_{\mathrm{NC}}$, and are:

$$
\begin{aligned}
& \left.\dot{v}_{\mathbf{N}}(t)\right|_{\text {coupling }}=-\frac{\pi}{\hbar^{2}} \int d^{3} \mathbf{x} \int d^{3} \mathbf{x}^{\prime}\left(2 \sum _ { 4 3 2 } \sum _ { m } \left\{\delta^{p}\left(\Delta \omega_{234}-\epsilon_{m}\right) Z_{432}(\mathbf{x}) Z_{432}^{\dagger}\left(\mathbf{x}^{\prime}\right) X_{m}^{\dagger}(\mathbf{x}) X_{m}\left(\mathbf{x}^{\prime}\right) v_{\mathbf{N}}(t)\right.\right. \\
& \left.+\delta^{p}\left(-\Delta \omega_{234}+\epsilon_{m}\right) v_{\mathbf{N}}(t) Z_{432}(\mathbf{x}) Z_{432}^{\dagger}\left(\mathbf{x}^{\prime}\right) X_{m}^{\dagger}(\mathbf{x}) X_{m}\left(\mathbf{x}^{\prime}\right)-2 \delta\left(\Delta \omega_{234}-\epsilon_{m}\right) X_{m}\left(\mathbf{x}^{\prime}\right) Z_{432}^{\dagger}\left(\mathbf{x}^{\prime}\right) v_{\mathbf{N}-\mathbf{e}_{432}}(t) Z_{432}(\mathbf{x}) X_{m}^{\dagger}(\mathbf{x})\right\} \\
& +2 \sum_{432} \sum_{m}\left\{\delta^{p}\left(-\Delta \omega_{234}+\epsilon_{m}\right) Z_{432}^{\dagger}(\mathbf{x}) Z_{432}\left(\mathbf{x}^{\prime}\right) X_{m}(\mathbf{x}) X_{m}^{\dagger}\left(\mathbf{x}^{\prime}\right) v_{\mathbf{N}}(t)\right.
\end{aligned}
$$




$$
\begin{aligned}
& \left.+\delta^{p}\left(\Delta \omega_{234}-\epsilon_{m}\right) v_{\mathbf{N}}(t) Z_{432}^{\dagger}(\mathbf{x}) Z_{432}\left(\mathbf{x}^{\prime}\right) X_{m}(\mathbf{x}) X_{m}^{\dagger}\left(\mathbf{x}^{\prime}\right)-2 \delta\left(\Delta \omega_{234}-\epsilon_{m}\right) X_{m}^{\dagger}\left(\mathbf{x}^{\prime}\right) Z_{432}\left(\mathbf{x}^{\prime}\right) v_{\mathbf{N}+\mathbf{e}_{432}}(t) Z_{432}^{\dagger}(\mathbf{x}) X_{m}(\mathbf{x})\right\} \\
& +4 \sum_{42} \sum_{r}\left\{\delta^{p}\left(\Delta \omega_{24}-\beta_{r}\right) Z_{42}(\mathbf{x}) Z_{42}^{\dagger}\left(\mathbf{x}^{\prime}\right) U_{r}^{\dagger}(\mathbf{x}) U_{r}\left(\mathbf{x}^{\prime}\right) v_{\mathbf{N}}(t)\right. \\
& \left.+\delta^{p}\left(-\Delta \omega_{24}+\beta_{r}\right) v_{\mathbf{N}}(t) Z_{42}(\mathbf{x}) Z_{42}^{\dagger}\left(\mathbf{x}^{\prime}\right) U_{r}^{\dagger}(\mathbf{x}) U_{r}\left(\mathbf{x}^{\prime}\right)-2 \delta\left(\Delta \omega_{24}-\beta_{r}\right) U_{r}\left(\mathbf{x}^{\prime}\right) Z_{42}^{\dagger}\left(\mathbf{x}^{\prime}\right) v_{\mathbf{N}-\mathbf{e}_{42}}(t) Z_{42}(\mathbf{x}) U_{r}^{\dagger}(\mathbf{x})\right\} \\
& +\sum_{4} \sum_{r}\left\{\delta^{p}\left(\omega_{4}-\beta_{r}^{\prime}\right) Z_{4}(\mathbf{x}) Z_{4}^{\dagger}\left(\mathbf{x}^{\prime}\right) V_{r}^{\dagger}(\mathbf{x}) V_{r}\left(\mathbf{x}^{\prime}\right) v_{\mathbf{N}}(t)\right. \\
& \left.+\delta^{p}\left(-\omega_{4}+\beta_{r}^{\prime}\right) v_{\mathbf{N}}(t) Z_{4}(\mathbf{x}) Z_{4}^{\dagger}\left(\mathbf{x}^{\prime}\right) V_{r}^{\dagger}(\mathbf{x}) V_{r}\left(\mathbf{x}^{\prime}\right)-2 \delta\left(\omega_{4}-\beta_{r}^{\prime}\right) V_{r}\left(\mathbf{x}^{\prime}\right) Z_{4}^{\dagger}\left(\mathbf{x}^{\prime}\right) v_{\mathbf{N}-\mathbf{e}_{4}}(t) Z_{4}(\mathbf{x}) V_{r}^{\dagger}(\mathbf{x})\right\} \\
& +\sum_{4} \sum_{r}\left\{\delta^{p}\left(-\omega_{4}+\beta_{r}^{\prime}\right) Z_{4}^{\dagger}(\mathbf{x}) Z_{4}\left(\mathbf{x}^{\prime}\right) V_{r}(\mathbf{x}) V_{r}^{\dagger}\left(\mathbf{x}^{\prime}\right) v_{\mathbf{N}}(t)\right. \\
& \left.\left.+\delta^{p}\left(\omega_{4}-\beta_{r}^{\prime}\right) v_{\mathbf{N}}(t) Z_{4}^{\dagger}(\mathbf{x}) Z_{4}\left(\mathbf{x}^{\prime}\right) V_{r}(\mathbf{x}) V_{r}^{\dagger}\left(\mathbf{x}^{\prime}\right)-2 \delta\left(\omega_{4}-\beta_{r}^{\prime}\right) V_{r}^{\dagger}\left(\mathbf{x}^{\prime}\right) Z_{4}\left(\mathbf{x}^{\prime}\right) v_{\mathbf{N}+\mathbf{e}_{4}}(t) Z_{4}^{\dagger}(\mathbf{x}) V_{r}(\mathbf{x})\right\}\right)
\end{aligned}
$$

These expressions use the function

$$
\delta^{p}(\omega)=\delta(\omega)-\frac{i}{\pi} \frac{\mathrm{P}}{\omega} .
$$

However, for simplicity we shall usually neglect the dispersive effects which arise from the principal valu integral by setting $\delta^{p} \rightarrow \delta$ everywhere.

\section{Full master equation}

The full density operator, in the approximation being used for these equations of motion, can be written as

$$
\rho(t)=\sum_{\mathbf{N}} v_{\mathbf{N}}(t)
$$

where the summation is over all $\mathbf{N}$ in the non-condensate band. Summing over $\mathbf{N}$ in 3.36 , 3.38a 3.38d, and in 3.43a $3.43 \mathrm{e}$, it is easy to see that one obtains an equation for $\rho(t)$ which is of exactly the same form, but in which $v_{\mathbf{N}}(t), v_{\mathbf{N}-\mathbf{e}}(t) \rightarrow \rho(t)$ for any $\mathbf{N}, \mathbf{e}$.

\section{INTERPRETATION OF THE MASTER EQUATION}

The master equation gives a representation of transitions due to collisions between states, defined by wavelets in the non-condensate-band, or eigenfunctions in the condensate band in their respective effective potentials which already include the majority of mean-field effects. The collisional terms include those purely within the non-condensate-band as in $(3.38 \mathrm{~d})$, and those which give rise to energy and matter transfer between the two bands, as in $3.43 \mathrm{~b}$ 3.43e. In addition to the truly irreversible collisional terms, there are also terms which arise from principal value integrals, which cause energy level shifts, since they take the form of a commutator. These terms are explicit in (3.38c), and are implicit in the $\delta^{P}$ terms in (3.43b-3.43e, and are analogous to the Lamb and Stark shift terms which arise similarly in quantum optics. They are equivalent to energy level shifts found by using second order perturbation theory.

\section{A. Stationary solutions of the master equation}

The residual mean-field terms and the level shifts are not expected to be large, nor do they in any way change the essential picture of transitions. To the extent that we can neglect these terms, the quantum kinetic master equation in the form 3.35) conserves

$$
\begin{aligned}
H_{a}^{\mathrm{eff}}+H_{\mathrm{C}}^{\mathrm{eff}} & \equiv H_{\mathrm{tot}}^{\mathrm{eff}} \\
N_{\mathrm{NC}}+N_{\mathrm{C}} & =N,
\end{aligned}
$$

so that any function of these provides a stationary solution. The grand canonical stationary solution takes the form

$$
\rho \propto \exp \left(-\frac{H_{a}^{\mathrm{eff}}-\mu N_{\mathrm{NC}}}{k T}\right) \otimes \exp \left(-\frac{H_{\mathrm{C}}^{\mathrm{eff}}-\mu N_{\mathrm{C}}}{k T}\right) .
$$

but the fact that $N_{\mathrm{NC}}+N_{\mathrm{C}}$ is conserved means that the restriction of the grand canonical form to any fixed value of $N_{\mathrm{NC}}+N_{\mathrm{C}}$ must also be a solution. The correlation functions of the non-condensate operators are quite straightforward

$$
\left\langle A_{\mathbf{Q}, \mathbf{r}}^{\dagger} A_{\mathbf{Q}^{\prime}, \mathbf{r}^{\prime}}\right\rangle=\delta_{\mathbf{r}, \mathbf{r}^{\prime}} \delta_{\mathbf{Q}, \mathbf{Q}^{\prime}} \bar{N}_{T}(\mathbf{Q})
$$

with 


$$
\begin{aligned}
\bar{N}_{T}(\mathbf{Q}) & =\left[\exp \left(\frac{E(\mathbf{Q})-\mu}{k T}\right)-1\right]^{-1} \\
\approx & {\left[\exp \left(\frac{\hbar^{2} \mathbf{K}(\mathbf{Q}, \mathbf{r})^{2} / 2 m+V_{\mathrm{NC}}^{\mathrm{eff}}(\mathbf{r})-\mu}{k T}\right)-1\right]^{-1} . }
\end{aligned}
$$

We can also deduce that the stationary field correlation functions can be written

$$
\begin{aligned}
& \left\langle\psi_{\mathbf{Q}}^{\dagger}(\mathbf{x}) \psi_{\mathbf{Q}^{\prime}}\left(\mathbf{x}^{\prime}\right)\right\rangle \\
& \quad=\delta_{\mathbf{Q}, \mathbf{Q}^{\prime}} \bar{N}_{T}(\mathbf{Q}) \sum_{\mathbf{r}} w_{\mathbf{K}(\mathbf{Q}, \mathbf{r}), \mathbf{r}}(\mathbf{x}) w_{\mathbf{K}(\mathbf{Q}, \mathbf{r}), \mathbf{r}}^{*}\left(\mathbf{x}^{\prime}\right) \\
& \quad \approx \delta_{\mathbf{Q}, \mathbf{Q}^{\prime}} \bar{N}_{T}(\mathbf{Q}) e^{i \mathbf{K}\left(\mathbf{Q}, \frac{\mathbf{x}+\mathbf{x}^{\prime}}{2}\right) \cdot\left(\mathbf{x}-\mathbf{x}^{\prime}\right)} g_{\mathbf{Q}}\left(\mathbf{x}, \mathbf{x}^{\prime}\right),
\end{aligned}
$$

where the last line comes from the asymptotic form (2.12). (These forms will also be valid to a very good degree of approximation even in a canonical ensemble formulation in which $N_{\mathrm{NC}}$ is fixed.)

\section{B. Relationship to the Hartree-Fock-Bogoliubov method}

The inclusion of the mean-field terms defines the stationary solutions of the Hamiltonian part in a form which is closely related to the Hartree-Fock-Bogoliubov method [20]. The task which must be accomplished here is the construction of best estimates of the condensate-band density $\bar{\rho}(\mathbf{x})$ and of the noncondensate-band density $\bar{n}(\mathbf{x})$ as defined in Sect. II B 3.

\section{Non-condensate-band density $\bar{n}(\mathbf{x})$}

The non-condensate-band density must be chosen in the form which takes account of the projected form of the interaction in 3.14, i.e.,

$$
\bar{n}(\mathbf{x})=\sum_{\mathbf{Q} \in R_{\mathrm{NC}}}\left\langle\psi_{\mathbf{Q}}^{\dagger}(\mathbf{x}) \psi_{\mathbf{Q}}(\mathbf{x})\right\rangle .
$$

and when we are dealing with the stationary state, this is given through 4.8 by as

$$
\begin{aligned}
& \bar{n}(\mathbf{x})=\sum_{\mathbf{Q} \in R_{\mathrm{NC}}} \bar{N}_{T}(\mathbf{Q}) g_{\mathbf{Q}}(\mathbf{x}, \mathbf{x}) \\
& \quad \approx \int_{E(\mathbf{K}, \mathbf{x})>E_{R}} d^{3} \mathbf{K}\left[\exp \left(\frac{E(\mathbf{K}, \mathbf{x})-\mu}{k T}\right)-1\right]^{-1} .
\end{aligned}
$$

Here

$$
\begin{aligned}
E(\mathbf{K}, \mathbf{x}) & =\frac{\hbar^{2} \mathbf{K}^{2}}{2 m}+V_{\mathrm{NC}}^{\mathrm{eff}}(\mathbf{x}) \\
& =\frac{\hbar^{2} \mathbf{K}^{2}}{2 m}+V_{T}(\mathbf{x})+2 u(\bar{\rho}(\mathbf{x})+\bar{n}(\mathbf{x})) .
\end{aligned}
$$

This amounts to a local density approximation for the atom density of the non-condensate-band.

\section{Condensate-band density $\bar{\rho}(\mathbf{x})$}

It is now necessary to specify $\bar{\rho}(\mathbf{x})$, which means we need to know the condensate state. From (4.3) we see that this requires the diagonalization of $H_{\mathrm{C}}^{\mathrm{eff}}-\mu N_{\mathrm{C}}$, where $H_{\mathrm{C}}^{\text {eff }}$ is given by (2.37). This can be done approximately by using the Bogoliubov method [⿰氵⿹丁口⿹丁口 , generalized to its number conserving form as given in [7], but using the $V_{\mathrm{C}}^{\text {eff }}(\mathbf{x})$ potential, which includes the mean field terms arising from the non-condensateband. This would mean that we write

$$
\phi(\mathbf{x}) \approx A\left(\xi_{N_{\mathrm{C}}}(\mathbf{x})+\frac{1}{\sqrt{N_{\mathrm{C}}+1}} \chi(\mathbf{x})\right),
$$

where the operator $A$ is a destruction operator for the number of atoms in the condensate-band, so $A^{\dagger} A$ has the eigenvalue $N_{\mathrm{C}}$, and $\xi_{N_{\mathrm{C}}}(\mathbf{x})$ is a solution of the Gross-Pitaevskii equation for $N_{\mathrm{C}}$ particles in the effective potential. Thus

$$
-\frac{\hbar^{2} \nabla^{2}}{2 m} \xi_{N_{\mathrm{C}}}(\mathbf{x})+V_{\mathrm{C}}^{\mathrm{eff}}(\mathbf{x}) \xi_{N_{\mathrm{C}}}(\mathbf{x})+u N_{\mathrm{C}}\left|\xi_{N_{\mathrm{C}}}(\mathbf{x})\right|^{2} \xi_{N_{\mathrm{C}}}(\mathbf{x})=\mu_{N_{\mathrm{C}}} \xi_{N_{\mathrm{C}}}(\mathbf{x}) .
$$

The residual part $\chi(\mathbf{x})$ is determined in terms of quasiparticles, thus

$$
\chi(\mathbf{x})=\sum_{m}\left[p_{m}(\mathbf{x}) b_{m}+q_{m}(\mathbf{x}) b_{m}^{\dagger}\right]
$$

where $p_{m}(\mathbf{x})$ and $q_{m}(\mathbf{x})$ diagonalize the residual Hamiltonian

$$
\begin{aligned}
\mathcal{H}_{3} & =-\frac{\hbar^{2}}{2 m} \int d^{3} \mathbf{x} \chi^{\dagger}(\mathbf{x}) \nabla^{2} \chi(\mathbf{x})+\int d^{3} \mathbf{x} \chi^{\dagger}(\mathbf{x}) V_{\mathrm{C}}^{\mathrm{eff}}(\mathbf{x}) \chi(\mathbf{x}) \\
& +\int d^{3} \mathbf{x}\left\{\frac{u N_{\mathrm{C}}}{2}\left(\xi_{N_{\mathrm{C}}}(\mathbf{x}) \chi^{\dagger}(\mathbf{x})\right)^{2}+\frac{u N_{\mathrm{C}}}{2}\left(\xi_{N_{\mathrm{C}}}^{*}(\mathbf{x}) \chi(\mathbf{x})\right)^{2}+\chi^{\dagger}(\mathbf{x}) \chi(\mathbf{x})\left(2 u N_{\mathrm{C}}\left|\xi_{N_{\mathrm{C}}}(\mathbf{x})\right|^{2}-\mu_{N_{\mathrm{C}}}\right)\right\} \\
& -\frac{u N_{\mathrm{C}}}{2} \int d^{3} \mathbf{y}\left|\xi_{N_{\mathrm{C}}}(\mathbf{y})\right|^{4} .
\end{aligned}
$$


The diagonalization of $\mathcal{H}_{3}$ is equivalent to solving the Bogoliubov-de Gennes equations [26] with a potential $V_{\mathrm{C}}^{\text {eff }}(\mathbf{x})$, and with an upper cutoff energy equal to $E_{R}-\mu_{N_{\mathrm{C}}}$. The wavefunctions $p_{m}(\mathbf{x})$ and $q_{m}(\mathbf{x})$ are the projections of the resulting solutions onto the subspace orthogonal to $\xi_{N_{\mathrm{C}}}(\mathbf{x})$.

This procedure gives solutions with any number $N_{\mathrm{C}}, N_{\mathrm{NC}}$ of condensate- or non-condensate-band particles. The value of the $\bar{\rho}(\mathbf{x})$ which arises is then

$$
\begin{aligned}
\bar{\rho}(\mathbf{x}) \approx & N_{\mathrm{C}}\left|\xi_{N_{C}}(\mathbf{x})\right|^{2} \\
& +\sum_{m}\left\{n_{m}\left|p_{m}(\mathbf{x})\right|^{2}+\left(n_{m}+1\right)\left|q_{m}(\mathbf{x})\right|^{2}\right\} .
\end{aligned}
$$

\section{Validity of the method}

1. The Bogoliubov approximation used here will be valid when the majority of the atoms in the condensate-band are in fact in the condensate level. This is not normally the majority of the atoms in the system, since there may be a very large number of atoms in the non-condensate-band, which are treated essentially by a local density approximation.

2. The values of $\bar{n}(\mathbf{x})$ and $\bar{\rho}(\mathbf{x})$ are used then to determine the mean field potentials, and hence the appropriate energy levels which provide a basis within which the master equation now describes time evolution, including transfer of energy and atoms from one band to the other, as well as the residual reversible processes.

3. The procedure for determining this basis is essentially equivalent to the Hartree-Fock-Bogoliubov method in the Popov approximation, but only in its time independent form.

4. Note that the chemical potentials and temperatures of the stationary solutions of the construction so far used can be determined independently of each other-it is only when we in- clude the master equation coupling terms that the condensateband and non-condensate-band are forced to evolve to an equilibrium in which the chemical potentials and temperatures are the same. The relevant equations of motion can then be computed in various degrees of simplification, and it is these that should be compared with the time-dependent Hartree-FockBogoliubov equations of motion.

5. The energy levels, wavefunctions and densities given by this process are to be seen as a way of choosing a good basis in terms of which the processes described by the master equation (3.35) can be described. As well as the irreversible processes, there will be corrections to these which also arise from the master equation, and which can be in principle computed.

\section{TIME-INDEPENDENT LOCAL EQUILIBRIUM APPROXIMATION}

In this section we will show how the methodology of QKIII arises. We assume that the scattering in $R_{\mathrm{NC}}$ is strong, and we are only interested in the behavior of the condensate and the modes in $R_{\mathrm{C}}$. We then factorize the total density operator into a quasi-thermalized non-condensate part $\rho_{B}$, and a density operator $\rho_{\mathrm{C}}(t)$, which describes $R_{\mathrm{C}}$. Thus

$$
\rho(t)=\sum_{\mathbf{N}} v_{\mathbf{N}}(t) \rightarrow \rho_{B} \otimes \rho_{\mathrm{C}}(t)
$$

and $\rho_{B}$ is of course time-independent. We use a local equilibrium approximation, in which $\rho_{B}$ is such that the averages of the products of field operators in $R_{\mathrm{NC}}$ are quasi-thermal, in much the same way as was used in the derivation of the Uehling-Uhlenbeck equation in QKI. Thus we write, as in (4.8)

$$
\left\langle\psi_{\mathbf{Q}}^{\dagger}(\mathbf{x}) \psi_{\mathbf{Q}^{\prime}}\left(\mathbf{x}^{\prime}\right)\right\rangle=\delta_{\mathbf{Q}, \mathbf{Q}^{\prime}} e^{i \mathbf{K}\left(\mathbf{Q}, \frac{\mathbf{x}+\mathbf{x}^{\prime}}{2}\right) \cdot\left(\mathbf{x}-\mathbf{x}^{\prime}\right)} g_{\mathbf{Q}}\left(\mathbf{x}-\mathbf{x}^{\prime}\right) F_{\mathbf{Q}}\left(\frac{\mathbf{x}+\mathbf{x}^{\prime}}{2}\right) .
$$

This allows for thermalization locally, in contrast to 4.8$)$, for which $F_{\mathbf{Q}}\left(\frac{\mathbf{x}+\mathbf{x}^{\prime}}{2}\right) \rightarrow \bar{N}_{T}(\mathbf{Q})$ which give a global thermalization. We use the Gaussian factorization properties of the the averages of the products of more than two field operators, so that for example, we can write

$$
\begin{aligned}
\left\langle\psi_{\mathbf{Q}_{1}}^{\dagger}(\mathbf{x}) \psi_{\mathbf{Q}_{2}}^{\dagger}(\mathbf{x}) \psi_{\mathbf{Q}_{1}^{\prime}}\left(\mathbf{x}^{\prime}\right) \psi_{\mathbf{Q}_{2}^{\prime}}\left(\mathbf{x}^{\prime}\right)\right\rangle= & \left(\delta_{\mathbf{Q}_{1}, \mathbf{Q}_{1}^{\prime}} \delta_{\mathbf{Q}_{2}, \mathbf{Q}_{2}^{\prime}}+\delta_{\mathbf{Q}_{1}, \mathbf{Q}_{2}^{\prime}} \delta_{\mathbf{Q}_{2}, \mathbf{Q}_{1}^{\prime}}\right) g_{\mathbf{Q}}\left(\mathbf{x}-\mathbf{x}^{\prime}\right)^{2} F_{\mathbf{Q}_{1}}\left(\frac{\mathbf{x}+\mathbf{x}^{\prime}}{2}\right) F_{\mathbf{Q}_{2}}\left(\frac{\mathbf{x}+\mathbf{x}^{\prime}}{2}\right) \\
& \times e^{\left\{i \mathbf{K}\left(\mathbf{Q}_{1}, \frac{\mathbf{x}+\mathbf{x}^{\prime}}{2}\right)+i \mathbf{K}\left(\mathbf{Q}_{2}, \frac{\mathbf{x}+\mathbf{x}^{\prime}}{2}\right)\right\} \cdot\left(\mathbf{x}-\mathbf{x}^{\prime}\right)}
\end{aligned}
$$

\section{A. Master equation}

Tracing over the non-condensate modes, we get

$$
\dot{\rho}_{\mathrm{C}}(t)=-\frac{i}{\hbar} \int d^{3} \mathbf{x}\left[\phi^{\dagger}(\mathbf{x})\left(-\frac{\hbar^{2} \nabla^{2}}{2 m}+V_{\mathrm{C}}^{\mathrm{eff}}(\mathbf{x})+\frac{1}{2} u \phi^{\dagger}(\mathbf{x}) \phi(\mathbf{x})\right) \phi(\mathbf{x}), \rho_{\mathrm{C}}\right]
$$




$$
\begin{aligned}
+\int d^{3} \mathbf{x} \int d^{3} \mathbf{x}^{\prime}( & \sum_{m} G^{(+)}\left(\mathbf{x}-\mathbf{x}^{\prime}, \epsilon_{m}\right)\left(2 X_{m}(\mathbf{x}) \rho_{\mathrm{C}} X_{m}^{\dagger}\left(\mathbf{x}^{\prime}\right)-\rho_{\mathrm{C}} X_{m}^{\dagger}\left(\mathbf{x}^{\prime}\right) X_{m}(\mathbf{x})-X_{m}^{\dagger}\left(\mathbf{x}^{\prime}\right) X_{m}(\mathbf{x}) \rho_{\mathrm{C}}\right) \\
& +\sum_{m} G^{(-)}\left(\mathbf{x}-\mathbf{x}^{\prime}, \epsilon_{m}\right)\left(2 X_{m}^{\dagger}(\mathbf{x}) \rho_{\mathrm{C}} X_{m}\left(\mathbf{x}^{\prime}\right)-\rho_{\mathrm{C}} X_{m}\left(\mathbf{x}^{\prime}\right) X_{m}^{\dagger}(\mathbf{x})-X_{m}\left(\mathbf{x}^{\prime}\right) X_{m}^{\dagger}(\mathbf{x}) \rho_{\mathrm{C}}\right) \\
& +\sum_{r} M\left(\mathbf{x}-\mathbf{x}^{\prime}, \beta_{r}\right)\left(2 U_{r}(\mathbf{x}) \rho_{\mathrm{C}} U_{r}^{\dagger}\left(\mathbf{x}^{\prime}\right)-\rho_{\mathrm{C}} U_{r}^{\dagger}\left(\mathbf{x}^{\prime}\right) U_{r}(\mathbf{x})-U_{r}^{\dagger}\left(\mathbf{x}^{\prime}\right) U_{r}(\mathbf{x}) \rho_{\mathrm{C}}\right) \\
& +\sum_{r} E^{(+)}\left(\mathbf{x}-\mathbf{x}^{\prime}, \beta_{r}^{\prime}\right)\left(2 V_{r}(\mathbf{x}) \rho_{\mathrm{C}} V_{r}^{\dagger}\left(\mathbf{x}^{\prime}\right)-\rho_{\mathrm{C}} V_{r}^{\dagger}\left(\mathbf{x}^{\prime}\right) V_{r}(\mathbf{x})-V_{r}^{\dagger}\left(\mathbf{x}^{\prime}\right) V_{r}(\mathbf{x}) \rho_{\mathrm{C}}\right) \\
& \left.+\sum_{r} E^{(-)}\left(\mathbf{x}-\mathbf{x}^{\prime}, \beta_{r}^{\prime}\right)\left(2 V_{r}^{\dagger}(\mathbf{x}) \rho_{\mathrm{C}} V_{r}\left(\mathbf{x}^{\prime}\right)-\rho_{\mathrm{C}} V_{r}\left(\mathbf{x}^{\prime}\right) V_{r}^{\dagger}(\mathbf{x})-V_{r}\left(\mathbf{x}^{\prime}\right) V_{r}^{\dagger}(\mathbf{x}) \rho_{\mathrm{C}}\right)\right)
\end{aligned}
$$

in which the quantities $E^{( \pm)}, M, G^{( \pm)}$are given by

$$
\begin{aligned}
G^{(+)}\left(\mathbf{x}-\mathbf{x}^{\prime}, \omega\right) & =\frac{2 \pi}{\hbar^{2}} \operatorname{Tr}_{B}\left\{\sum_{123} \delta\left(\omega_{1}+\omega_{2}-\omega_{3}-\omega\right) Z_{123}^{\dagger}\left(\mathbf{x}^{\prime}\right) Z_{123}(\mathbf{x}) \rho_{B}\right\} \\
G^{(-)}\left(\mathbf{x}-\mathbf{x}^{\prime}, \omega\right) & =\frac{2 \pi}{\hbar^{2}} \operatorname{Tr}_{B}\left\{\sum_{123} \delta\left(\omega_{1}+\omega_{2}-\omega_{3}-\omega\right) Z_{123}(\mathbf{x}) Z_{123}^{\dagger}\left(\mathbf{x}^{\prime}\right) \rho_{B}\right\} \\
M\left(\mathbf{x}-\mathbf{x}^{\prime}, \omega\right) & =\frac{4 \pi}{\hbar^{2}} \operatorname{Tr}_{B}\left\{\sum_{12} \delta\left(\omega_{1}-\omega_{2}-\omega\right) Z_{12}^{\dagger}\left(\mathbf{x}^{\prime}\right) Z_{12}(\mathbf{x}) \rho_{B}\right\} \\
E^{(+)}\left(\mathbf{x}-\mathbf{x}^{\prime}, \omega\right) & =\frac{\pi}{\hbar^{2}} \operatorname{Tr}_{B}\left\{\sum_{1} \delta\left(\omega_{1}-\omega\right) Z_{1}^{\dagger}\left(\mathbf{x}^{\prime}\right) Z_{1}(\mathbf{x}) \rho_{B}\right\} \\
E^{(-)}\left(\mathbf{x}-\mathbf{x}^{\prime}, \omega\right) & =\frac{\pi}{\hbar^{2}} \operatorname{Tr}_{B}\left\{\sum_{1} \delta\left(\omega_{1}-\omega\right) Z_{1}(\mathbf{x}) Z_{1}^{\dagger}\left(\mathbf{x}^{\prime}\right) \rho_{B}\right\} .
\end{aligned}
$$

The master equation $5.4 \mathrm{a}-5.4 \mathrm{f}$ is of the same form as given in QKIII, apart from

1. The expression of the non-condensate band summations in terms of Q-bands, rather than the continuum approximation chosen in QKIII as integrals over $\mathbf{K}$ variables. In this degree of approximation, this leads only to a change in the range of $\mathbf{K}$ as a function of $\mathbf{x}$.

2. The potential defining the $\mathbf{Q}$-bands is the effective potential $V_{\mathrm{NC}}^{\mathrm{eff}}(\mathrm{x})$, which depends on the occupation of the condensateband, rather than simply the trapping potential as in QKIII.

\section{B. Interactions between quasiparticles}

As written in (3.37) and (5.4a) the condensate-band Hamiltonian is treated exactly. Using the Bogoliubov approximation, we can approximate the condensate-band Hamiltonian term (5.4a) in the form

$$
H=\sum_{m} \epsilon_{m} b^{\dagger} b_{m}+E_{0}(N) .
$$

Although the irreversible terms in (5.4a 5.41 give damping and thermalization, this approximation does omit the effect of the higher order terms, which can generate scattering of the quasiparticles by quasiparticles. Graham [27] has pointed out that these can be significant, even though the quasiparticle density of states is rather low, essentially because the very low energy quasiparticles involve very large numbers of atoms. If this pertains, we have three possibilities.

1) We can treat the quasiparticles in essentially the same manner as the particles in the non-condensate band; that is, we can assume they are thermalized, and compute scattering rates. There is no need, however, to use wavelets, since we can simply use the discrete quasiparticle states, of which there should be relatively few compared to the non-condensate band states. This would correspond to Graham's methodology.

2) It is possible that an exact treatment of the condensate band Hamiltonian could be feasible, provided it is realistic to take this as consisting of only a relatively few quasiparticle levels. Stringari [25] has pointed out that in fact in the case of a trap, very few of the levels have any genuine phonon-like character. This could be done in combination with the use of a stochastic wavefunction method for the interactions with the non-condensate band.

3) As an intermediate form of approximation, it could well be feasible to use a positive-P-representation methodology, rather like that used by Drummond and Corney [28] for the condensate-band Hamiltonian in a quasiparticle basis. 
We shall leave further developments of this aspect to another publication

\section{TIME DEPENDENT LOCAL EQUILIBRIUM APPROXIMATION}

The local equilibrium approximation, when applied to the quantum-kinetic master equation of QKI was shown to give rise to the well known Uehling-Uhlenbeck equation [18]. A similar method applied to this case leads to a description of the coupling of thermalized vapor to the condensate. Together with the equations of motion for the condensate density operator as in Sec. III.D.3 of QKIII, or the rate equation approximations to these, this gives a description of the coupled motion of the condensate and non-condensate. From this description one can take further limits to get coupled equations of motion in the hydrodynamic limit, or in the weak-collision limit.

To be precise, we define

$$
f_{\mathbf{K}}(\mathbf{x}) \equiv F_{\mathbf{Q}(\mathbf{K}, \mathbf{x})}(\mathbf{x})
$$

where $F$ is as defined in (5.2). (This is the same as in QKI). Using the same procedures as in QKI, we can eventually arrive at the equation of motion:

$$
\begin{aligned}
\frac{\partial f_{\mathbf{K}}(\mathbf{x})}{\partial t}= & \left.\frac{\partial f_{\mathbf{K}}(\mathbf{x})}{\partial t}\right|_{\mathrm{UU}}+\left.\frac{\partial f_{\mathbf{K}}(\mathbf{x})}{\partial t}\right|_{\mathrm{mf}} \\
& +\left.\frac{\partial f_{\mathbf{K}}(\mathbf{x})}{\partial t}\right|_{1}+\left.\frac{\partial f_{\mathbf{K}}(\mathbf{x})}{\partial t}\right|_{2}+\left.\frac{\partial f_{\mathbf{K}}(\mathbf{x})}{\partial t}\right|_{3}
\end{aligned}
$$

Of these, the first two terms represent flow and collisions of atoms in the non-condensate-band, and the last three are irreversible master equation terms, which represent the transfer of energy and atoms between the condensate-band and the noncondensate-band.
The equation of motion 6.2 depends on the condensateband state through the second term, which depends on the mean field determined by the mean condensate density $\rho(\mathbf{x}, t)$, as defined by (6.8). Thus we must complement 6.2 with an appropriate equation of motion for the condensate. At the simplest, we can simply use rate equations for the condensate level occupationsand compute the condensate density from (4.18). The condensate equations of motion should be written in terms of the time-independent basis set as in (4.16), which uses the time-independent mean-field potential $V_{\mathrm{C}}^{\text {eff }}(\mathbf{x})$. The deviation from the average density $n(\mathbf{x}, t)$ induces the additional Hamiltonian term (3.41,3.42, which can also be expressed in the Bogoliubov basis.

The process of condensate growth gives rise to an apparent time-dependence as follows. The basis set of states is different for every pair of values $N_{\mathrm{NC}}, N_{\mathrm{C}}$-thus the set $N_{\mathrm{NC}}, N_{\mathrm{C}}$ can be regarded as defining a set of independent sectors, each with its own basis states. When the coupling terms between the condensate-band and the non-condensate band are taken into account, the effect is to transfer the system between sectors by the transitions $N_{\mathrm{NC}}, N_{\mathrm{C}} \rightarrow N_{\mathrm{NC}} \pm 1, N_{\mathrm{C}} \mp 1$, and to provide a new basis for the density operator. However this can be accounted by a slight modification to the Bogoliubov expansion - thus this apparent time dependence can in fact be fully accounted for by the existing procedures.

\section{A. Collisions and flow in the non-condensate}

\section{Uehling-Uhlenbeck term}

This term is the part arising from streaming terms and collisions between atoms in the non-condensate-band with each other, and takes the form

$$
\begin{aligned}
\left.\frac{\partial f_{\mathbf{K}}(\mathbf{x})}{\partial t}\right|_{\mathrm{UU}}= & \left(\frac{\hbar \mathbf{K} \cdot \nabla_{\mathbf{x}}}{m}-\frac{\nabla_{\mathbf{x}} V_{\mathrm{NC}}^{\mathrm{eff}}(\mathbf{x}) \cdot \nabla_{\mathbf{K}}}{\hbar}\right) f_{\mathbf{K}}(\mathbf{x}) \\
& +\frac{2|u|^{2}}{h^{2}} \int_{R_{\mathrm{NC}}} d^{3} \mathbf{K}_{2} \int_{R_{\mathrm{NC}}} d^{3} \mathbf{K}_{3} \int_{R_{\mathrm{NC}}} d^{3} \mathbf{K}_{4} \delta\left(\mathbf{K}+\mathbf{K}_{2}-\mathbf{K}_{3}-\mathbf{K}_{4}\right) \delta\left(\omega+\omega_{2}-\omega_{3}-\omega_{4}\right) \\
& \times\left\{f_{\mathbf{K}}(\mathbf{x}) f_{\mathbf{K}_{2}}(\mathbf{x})\left[f_{\mathbf{K}_{3}}(\mathbf{x})+1\right]\left[f_{\mathbf{K}_{4}}(\mathbf{x})+1\right]-\left[f_{\mathbf{K}}(\mathbf{x})+1\right]\left[f_{\mathbf{K}_{2}}(\mathbf{x})+1\right] f_{\mathbf{K}_{3}}(\mathbf{x}) f_{\mathbf{K}_{4}}(\mathbf{x})\right\}
\end{aligned}
$$

Notice that the range of energies available is restricted to the condensate-band, which is consistent with the streaming term-thus there are no terms here which can transfer particles out of $R_{\mathrm{NC}}$.

\section{Mean-field corrections to the Uehling-Uhlenbeck term}

The Uehling-Uhlenbeck term includes only the flow of the non-condensate which arises as a result of the (timeindependent) effective potential $V_{\mathrm{NC}}^{\text {eff }}=2 u \bar{\rho}(\mathbf{x})+2 u \bar{n}(\mathbf{x})+$ $V_{T}(\mathbf{x})$. However, when the condensate is not timeindependent, the cancellation of the average values of $H_{\mathrm{mf}}$ as in 3.39, 3.42 does not occur, since $\bar{n}(\mathbf{x})$ and $\bar{\rho}(\mathbf{x})$ are taken as time independent estimates of the mean values. Including these in the equation of motion for $f_{\mathbf{K}}(\mathbf{x})$ gives rise to extra terms proportional to

$$
\left\langle\left[\int d^{3} \mathbf{y} \sum_{\mathbf{Q}^{\prime}} \psi_{\mathbf{Q}^{\prime}}^{\dagger}(\mathbf{y}) \psi_{\mathbf{Q}^{\prime}}(\mathbf{y}), \psi_{\mathbf{Q}}^{\dagger}(\mathbf{x}) \psi_{\mathbf{Q}}(\mathbf{x})\right]\right\rangle
$$




$$
\times\left\langle\sum_{\mathbf{Q}^{\prime \prime}} \psi_{\mathbf{Q}^{\prime \prime}}^{\dagger}(\mathbf{y}) \psi_{\mathbf{Q}^{\prime \prime}}(\mathbf{y})+\phi^{\dagger}(\mathbf{y}) \phi(\mathbf{y})-\bar{n}(\mathbf{y})-\bar{\rho}(\mathbf{y})\right\rangle .
$$

The commutator is non-zero only for $\mathbf{Q}^{\prime}=\mathbf{Q}$ since the commutators $\psi_{\mathbf{Q}}(\mathbf{x})$ etc. are non-local. If we use the form 5.2) to evaluate this average this commutator does vanish $-\mathrm{a}$ nonvanishing result only appears when we allow corrections to this as a result of the non-equilibrium state implicit in the situation. The detailed working is given in Appendix B; here we shall merely state the result, which is intuitively obvious, that for sufficiently smooth distributions in $\mathbf{Q}$ and $\mathbf{x}$, we find

$$
\begin{aligned}
& \left.\frac{\partial f_{\mathbf{K}}(\mathbf{x})}{\partial t}\right|_{\mathrm{mf}} \\
& =2 u \nabla_{\mathbf{x}}\{\bar{\rho}(\mathbf{x}, t)-\bar{\rho}(\mathbf{x})+\bar{n}(\mathbf{x}, t)-\bar{n}(\mathbf{x})\} \cdot \nabla_{\mathbf{K}} f_{\mathbf{K}}(\mathbf{x})
\end{aligned}
$$

where $\bar{n}(\mathbf{x}, t)$ and $\bar{\rho}(\mathbf{x}, t)$ are the time dependent versions of the condensate-band and non-condensate-band densities, i.e.,

$$
\begin{aligned}
\bar{n}(\mathbf{x}, t) & =\sum_{\mathbf{Q}^{\prime}} \operatorname{Tr}\left(\psi_{\mathbf{Q}^{\prime}}^{\dagger}(\mathbf{x}) \psi_{\mathbf{Q}^{\prime}}(\mathbf{x}) \bar{\rho}_{B}(t)\right) \\
& =\frac{1}{(2 \pi)^{3}} \int_{R_{\mathrm{NC}}} d^{3} \mathbf{K} f_{\mathbf{K}}(\mathbf{x}) . \\
\bar{\rho}(\mathbf{x}, t) & =\operatorname{Tr}\left(\phi^{\dagger}(\mathbf{x}) \phi(\mathbf{x}) \rho_{\mathrm{C}}(t)\right) .
\end{aligned}
$$

Thus the net effect on the Uehling-Uhlenbeck term is simply to make the replacement to the effective potential

$$
\begin{aligned}
V_{\mathrm{NC}}^{\mathrm{eff}}(\mathbf{x}) & \rightarrow V_{\mathrm{NC}}^{\mathrm{eff}}(\mathbf{x}, t) \\
& =V_{T}(\mathbf{x})+2 u \bar{\rho}(\mathbf{x}, t)+2 u \bar{n}(\mathbf{x}, t),
\end{aligned}
$$

that is, a time dependent version of the effective potential.

\section{Condensate growth term}

This is the principal term which gives rise to growth of the condensate. In the case that we use the Bogoliubov approximation to the condensate, and the notation of Sec.IId of QKIII, to write this in terms of the two rate functions

$$
\begin{gathered}
\operatorname{Rate}_{I}^{(+)}(\mathbf{K}, \mathbf{x}, \omega)=\frac{2 u^{2}}{(2 \pi)^{2} \hbar^{2}} \int_{R_{\mathrm{NC}}} d^{3} \mathbf{K}_{2} \int_{R_{\mathrm{NC}}} d^{3} \mathbf{K}_{4} \int d^{3} \mathbf{k} \delta\left(\Delta \omega_{2 \mathbf{K} 4}(\mathbf{x})-\omega\right) \delta\left(\mathbf{K}+\mathbf{K}_{4}-\mathbf{K}_{2}-\mathbf{k}\right) \\
\quad \times\left[1+f_{\mathbf{K}}(\mathbf{x})\right]\left[1+f_{4}(\mathbf{x})\right] f_{2}(\mathbf{x}) \mathcal{W}_{I}(\mathbf{x}, \mathbf{k}) \\
-\frac{u^{2}}{(2 \pi)^{2} \hbar^{2}} \int_{R_{\mathrm{NC}}} d^{3} \mathbf{K}_{3} \int_{R_{\mathrm{NC}}} d^{3} \mathbf{K}_{4} \int d^{3} \mathbf{k} \delta\left(\Delta \omega_{\mathbf{K} 34}(\mathbf{x})-\omega\right) \delta\left(\mathbf{K}_{3}+\mathbf{K}_{4}-\mathbf{K}-\mathbf{k}\right) \\
\quad \times f_{\mathbf{K}}(\mathbf{x}) f_{2}(\mathbf{x})\left[1+f_{3}(\mathbf{x})\right] \mathcal{W}_{I}(\mathbf{x}, \mathbf{k}) \\
\operatorname{Rate}_{I}^{(-)}(\mathbf{K}, \mathbf{x}, \omega)=\frac{2 u^{2}}{(2 \pi)^{2} \hbar^{2}} \int_{R_{\mathrm{NC}}} d^{3} \mathbf{K}_{2} \int_{R_{\mathrm{NC}}} d^{3} \mathbf{K}_{4} \int d^{3} \mathbf{k} \delta\left(\Delta \omega_{2 \mathbf{K} 4}(\mathbf{x})-\omega\right) \delta\left(\mathbf{K}+\mathbf{K}_{4}-\mathbf{K}_{2}-\mathbf{k}\right) \\
\times f_{\mathbf{K}}(\mathbf{x}) f_{4}(\mathbf{x})\left[1+f_{2}(\mathbf{x})\right] \mathcal{W}_{I}(\mathbf{x}, \mathbf{k}) \\
-\frac{u^{2}}{(2 \pi)^{2} \hbar^{2}} \int_{R_{\mathrm{NC}}} d^{3} \mathbf{K}_{3} \int_{R_{\mathrm{NC}}} d^{3} \mathbf{K}_{4} \int d^{3} \mathbf{k} \delta\left(\Delta \omega_{\mathbf{K} 34}(\mathbf{x})-\omega\right) \delta\left(\mathbf{K}_{3}+\mathbf{K}_{4}-\mathbf{K}-\mathbf{k}\right) \\
\times\left[1+f_{\mathbf{K}}(\mathbf{x})\right]\left[1+f_{2}(\mathbf{x})\right] f_{3}(\mathbf{x}) \mathcal{W}_{I}(\mathbf{x}, \mathbf{k})
\end{gathered}
$$

The term is then defined as

$$
\begin{aligned}
\left.\frac{\partial f(\mathbf{K}, \mathbf{x})}{\partial t}\right|_{1}= & N_{\mathrm{C}} \operatorname{Rate}_{0}^{(-)}\left(\mathbf{K}, \mathbf{x}, \mu_{N_{\mathrm{C}}} / \hbar\right)-\left(N_{\mathrm{C}}+1\right) \operatorname{Rate}_{0}^{(+)}\left(\mathbf{K}, \mathbf{x}, \mu_{N_{\mathrm{C}}} / \hbar\right) \\
& +\sum_{m}\left\{n_{m} \operatorname{Rate}_{m}^{(-)}\left(\mathbf{K}, \mathbf{x},\left(\mu_{N_{\mathrm{C}}}+\epsilon_{N_{\mathrm{C}}}^{m}\right) / \hbar\right)-\left(n_{m}+1\right) \operatorname{Rate}_{m}^{(+)}\left(\mathbf{K}, \mathbf{x},\left(\mu_{N_{\mathrm{C}}}+\epsilon_{N_{\mathrm{C}}}^{m}\right) / \hbar\right)\right. \\
& \left.+\left(n_{m}+1\right) \operatorname{Rate}_{m}^{(-)}\left(\mathbf{K}, \mathbf{x},\left(\mu_{N_{\mathrm{C}}}-\epsilon_{N_{\mathrm{C}}}^{m}\right) / \hbar\right)-n_{m} \operatorname{Rate}_{m}^{(+)}\left(\mathbf{K}, \mathbf{x},\left(\mu_{N_{\mathrm{C}}}-\epsilon_{N_{\mathrm{C}}}^{m}\right) / \hbar\right)\right\}
\end{aligned}
$$




\section{Scattering terms}

We also need an abbreviated notation for the number operators of the condensate-band

$$
\begin{aligned}
\tilde{n}_{I} & =\left\langle S_{I}^{\dagger} S_{I}\right\rangle \\
\tilde{m}_{I} & =\left\langle S_{I} S_{I}^{\dagger}\right\rangle,
\end{aligned}
$$

and in particular this means

$$
\begin{aligned}
\tilde{n}_{0} & =N_{\mathrm{C}} \\
\tilde{m}_{0} & =N_{\mathrm{C}}+1 \\
\tilde{n}_{m} & =N_{\mathrm{C}} n_{m} \\
\tilde{m}_{m} & =\left(N_{\mathrm{C}}+1\right)\left(n_{m}+1\right) \\
\tilde{n}_{-m} & =N_{\mathrm{C}}\left(n_{m}+1\right) \\
\tilde{m}_{-m} & =\left(N_{\mathrm{C}}+1\right) n_{m}
\end{aligned}
$$

Here we can again define some rate functions in the form

$$
\begin{gathered}
\operatorname{Rate}_{I J}(\mathbf{K}, \mathbf{x}, \omega)=\frac{8 \pi u^{2}}{\hbar^{2}} \int_{R_{\mathrm{NC}}} d^{3} \mathbf{K}_{2} \int d^{3} \mathbf{k} \int d^{3} \mathbf{k}^{\prime} \delta\left(\Delta \omega_{2 \mathbf{K}}(\mathbf{x})-\omega\right) \delta\left(\mathbf{K}-\mathbf{K}_{2}-\mathbf{k}+\mathbf{k}^{\prime}\right) \\
\times\left[1+f_{\mathbf{K}}(\mathbf{x})\right] f_{2}(\mathbf{x}) \mathcal{W}_{I}(\mathbf{x}, \mathbf{k}) \mathcal{W}_{J}\left(\mathbf{x}, \mathbf{k}^{\prime}\right) \\
-\frac{8 \pi u^{2}}{\hbar^{2}} \int_{R_{\mathrm{NC}}} d^{3} \mathbf{K}_{4} \int d^{3} \mathbf{k} \int d^{3} \mathbf{k}^{\prime} \delta\left(\Delta \omega_{\mathbf{K} 4}(\mathbf{x})-\omega\right) \delta\left(\mathbf{K}_{4}-\mathbf{K}-\mathbf{k}+\mathbf{k}^{\prime}\right) \\
\times f_{\mathbf{K}}(\mathbf{x})\left[1+f_{4}(\mathbf{x})\right] \mathcal{W}_{I}(\mathbf{x}, \mathbf{k}) \mathcal{W}_{J}\left(\mathbf{x}, \mathbf{k}^{\prime}\right)
\end{gathered}
$$

The term is then defined as

$$
\begin{aligned}
& \left.\frac{\partial f(\mathbf{K}, \mathbf{x})}{\partial t}\right|_{2}=\sum_{I J} \operatorname{Rate}_{I J}\left(\mathbf{K}, \mathbf{x}, \Omega_{I}\left(N_{\mathrm{C}}\right)-\Omega_{J}\left(N_{\mathrm{C}}\right)\right) \tilde{n}_{I} \tilde{m}_{J} \\
& \text { 5. Three-quasiparticle terms } \\
& \operatorname{Rate}_{I J K}^{(+)}(\mathbf{K}, \mathbf{x}, \omega)=\frac{2 \pi u^{2}}{\hbar^{2}} \int d^{3} \mathbf{k} \int d^{3} \mathbf{k}^{\prime} \int d^{3} \mathbf{k}^{\prime \prime} \delta\left(\Delta \omega_{\mathbf{K}}(\mathbf{x})-\omega\right) \delta\left(\mathbf{K}-\mathbf{k}+\mathbf{k}^{\prime}+\mathbf{k}^{\prime}\right) \\
& \times\left[1+f_{\mathbf{K}}(\mathbf{x})\right] \mathcal{W}_{I}(\mathbf{x}, \mathbf{k}) \mathcal{W}_{J}\left(\mathbf{x}, \mathbf{k}^{\prime}\right) \mathcal{W}_{K}\left(\mathbf{x}, \mathbf{k}^{\prime}\right) \\
& \operatorname{Rate}_{I J K}^{(-)}(\mathbf{K}, \mathbf{x}, \omega)=\frac{2 \pi u^{2}}{\hbar^{2}} \int d^{3} \mathbf{k} \int d^{3} \mathbf{k}^{\prime} \int d^{3} \mathbf{k}^{\prime \prime} \delta\left(\Delta \omega_{\mathbf{K}}(\mathbf{x})-\omega\right) \delta\left(\mathbf{K}-\mathbf{k}+\mathbf{k}^{\prime}+\mathbf{k}^{\prime}\right) \\
& \times f_{\mathbf{K}}(\mathbf{x}) \mathcal{W}_{I}(\mathbf{x}, \mathbf{k}) \mathcal{W}_{J}\left(\mathbf{x}, \mathbf{k}^{\prime}\right) \mathcal{W}_{K}\left(\mathbf{x}, \mathbf{k}^{\prime}\right)
\end{aligned}
$$

The term is then defined as

$$
\begin{aligned}
\left.\frac{\partial f(\mathbf{K}, \mathbf{x})}{\partial t}\right|_{3}= & \sum_{I J K}\left\{\operatorname{Rate}_{I J}^{(+)}\left[\mathbf{K}, \mathbf{x}, \Omega_{K}\left(N_{\mathrm{C}}\right)+\Omega_{I}\left(N_{\mathrm{C}}\right)-\Omega_{J}\left(N_{\mathrm{C}}\right)\right] \tilde{n}_{I} \tilde{m}_{J} \tilde{m}_{K}\right. \\
& \left.+\operatorname{Rate}_{I J K}^{(-)}\left[\mathbf{K}, \mathbf{x}, \Omega_{K}\left(N_{\mathrm{C}}\right)+\Omega_{I}\left(N_{\mathrm{C}}\right)-\Omega_{J}\left(N_{\mathrm{C}}\right)\right] \tilde{m}_{I} \tilde{n}_{J} \tilde{n}_{K}\right\} .
\end{aligned}
$$


The various rate in the growth, scattering, and three- $\phi$ terms are compatible with the same rates as seen by the condensate, i.e., equations $(126,127,141,143,144)$ of QKIII, in the sense that the rate at which a process removes/adds a particle to the condensate-band matches exactly with the corresponding rate at which the process adds/removes a particle from the noncondensate-band.

\section{CONCLUSION}

The principal results, in the sense that they can be applied, are the master equations of the time-independent local equilibrium approximation, Sect.III, and of the time-dependent local-equilibrium approximation of Sect. $\mathrm{V}$. The first of these was derived in QKIII, but without the inclusion of mean-field effects. These are now included by means of the use of effective potentials, which account for the majority of such effects, giving a description which is quite realistic. The use of this picture-with some technical simplifications-gives the basis for the condensate growth methodology of [17], and this seems to agree quite well with experiment.

However, the assumption of a static non-condensate-band is not always reasonable, so the methodology of Sect. $\mathrm{V}$ to get the time dependent local equilibrium approximation is necessary. Fortunately, the basic modification is essentially straightforward. The equations are modified so that the noncondensate band evolves according to a quantum Boltzmann equation, and the content of Sect. $\mathrm{V}$ is how one includes from the point of view of the non-condensate-band equation of motion the correct way of treating the transfer of atoms and energy between it and the condensate band. The necessary modifications are given in (6.12, 6.22 6.25), are easy to understand, but have a complexity which arises from the mixture of particle creation and destruction operators which defines the quasiparticle creation operators - this complexity is intrinsic to the problem and therefore unavoidable.

The whole procedure has been developed in terms of the Bogoliubov picture of condensate-band atoms moving in the effective potential, which includes mean-field effects, and is essentially a kind of Hartree-Fock picture. Even though no explicit depiction of the scattering of quasiparticles by each other is given, this can be treated if required by one of the following options.

1. We can ignore these scattering effects entirely, when they are negligible compared to the scattering of quasiparticle by non-condensate-band atoms. This will certainly be the case if there is a very large proportion of thermal vapor.

2. Notice however that the condensate band Hamiltonian $H_{\mathrm{C}}^{\mathrm{eff}}$ as defined in 3.36) is never itself explicitly approximated by the Bogoliubov form in the master equations. It is only the transition rates induced by collisions which have been expressed using the Bogoliubov approximation. One could in principle solve the master equation using the full $H_{\mathrm{C}}^{\mathrm{eff}}$, and the nonlinear mixing induced by the nonlinearity must be equivalent to the collisions of quasiparticles, because of the presence of the randomization induced by the collisions with the vapor.
3. What seems simplest is a compromise-approximate $H_{\mathrm{C}}^{\mathrm{eff}}$ by the Bogoliubov form taken to the next highest order, which gives rise to Belyayev terms. In the $1 / \sqrt{N}$ expansion in which the Bogoliubov method is valid the next higher order terms must be quite small. This is possible because the the condensate band consists only of a small fraction of the levels available, and the quartic terms which appear non-negligible in a conventional Bogoliubov [29] method are taken account of her by the use of the effective potentials.

Thus the quantum kinetic methodology is complete. It provides a logical combination of kinetics, as given by the quantum Boltzmann equation, with the field aspect, as given by the Bogoliubov picture. It is likely to be as accurate as any experiment will require, and has already been used to give a quantitative picture of condensate growth [16, 17], consistent with experiment.

\section{APPENDIX A: CONSTRUCTION OF WAVELETS}

\section{One dimensional systems}

In this case the wavelets can be expressed directly in terms of trap energy eigenfunctions. Consider the one dimensional trapping Hamiltonian

$$
\begin{aligned}
H_{T} & =\frac{p^{2}}{2 M}+V(x) \\
& =\sum_{n} E_{n}|n\rangle\langle n|
\end{aligned}
$$

We relabel these states by dividing them into groups labeled by an index $N=0,1,2, \ldots$, so that there are $\Delta_{N}$ states in each group, and we can now label the states for a fixed $N$ by a subsidiary index $l$. Thevalue of the parameter $\Delta_{N}$ will be fixed in due course, to give a description of maximum convenience.

This means we write the states as $|N, l\rangle_{g}$, where $l=$ $1,2,3, \ldots, \Delta_{N}-1$, and now the energy of a given state is $E_{n(N, l)}$, with

$$
n(N, l)=l-1+\sum_{N^{\prime}=0}^{N-1} \Delta_{N^{\prime}}
$$

Using this relabeling, we define the basic wavelet states as

$$
|N, m\rangle=\frac{e^{-\pi i\left(\Delta_{N}+1\right)}}{\sqrt{\Delta_{N}}} \sum_{l=1}^{\Delta_{N}} e^{2 \pi i l m / \Delta_{N}}|N, l\rangle_{g},
$$

where $m=1,2,3, \ldots, \Delta_{N}$. 


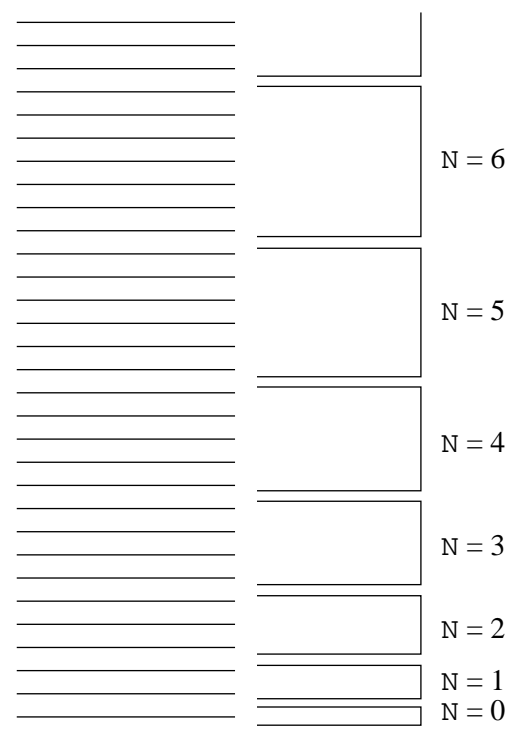

Fig.2. Grouping of Trap levels. Wavelets are formed by linear combinations of levels in each group.

These states form an orthonormal set

$$
\left\langle N^{\prime}, m^{\prime} \mid N, m\right\rangle=\delta_{N N^{\prime}} \delta_{m m^{\prime}}
$$

The actual wavelet functions are given by the position space representations of these states;

$$
w_{N, m}(x)=\langle x \mid N, m\rangle
$$

\section{a. Phase space variable $\theta$}

The best physical variable to use here is

$$
\theta \equiv 2 \pi m / \Delta_{N}
$$

This variable is an angle with the range

$$
\theta=\frac{2 \pi}{\Delta_{N}}, \frac{2 \times 2 \pi}{\Delta_{N}}, \frac{3 \times 2 \pi}{\Delta_{N}}, \ldots, 2 \pi
$$

This range approaches a continuum as $\Delta_{N} \rightarrow \infty$.

\section{b. Expression of the trapping Hamiltonian}

The average energy of the states $|N, l\rangle$ in the energy band $N$ is

$$
\bar{E}_{N}=\frac{1}{\Delta_{N}} \sum_{l=1}^{\Delta_{N}} E(N, l) .
$$

We can now write the trapping Hamiltonian $H_{T}$ as a part diagonal in both $N$ and $\theta$, and a residue which is diagonal in $N$ only;

$$
\begin{aligned}
& \left\langle N, \theta\left|H_{T}\right| N^{\prime}, \theta^{\prime}\right\rangle \\
& \quad=\delta_{N, N^{\prime}}\left\{\bar{E}_{N} \delta_{\theta \theta^{\prime}}+\left[\left\langle N, \theta\left|H_{T}\right| N^{\prime}, \theta^{\prime}\right\rangle-\bar{E}_{N} \delta_{\theta \theta^{\prime}}\right]\right\}
\end{aligned}
$$

It is now necessary to make some approximations in order to get some idea of the kinds of functions which will turn up. The major approximation is to assume the energy of each level is approximately a linear function of $l$ for any $N$. This is exact in the case of a harmonic potential.

Thus we write

$$
E(N, l)=\bar{E}(N)+\epsilon_{N}(l)
$$

where now, we approximate

$$
\epsilon_{N}(l) \approx \hbar \omega_{N}\left(l-\frac{\Delta_{N}+1}{2}\right),
$$

and this equation defines $\omega_{N}$. After some manipulation we find that

$$
\left\langle N, \theta\left|H_{T}\right| N^{\prime}, \theta^{\prime}\right\rangle=\delta_{N, N^{\prime}}\left\{\bar{E}_{N} \delta_{\theta \theta^{\prime}}-i \hbar \omega_{N} h_{N}\left(\theta^{\prime}-\theta\right)\right\} .
$$

Here $h_{N}(x)$ is defined by

$$
h_{N}(x)=\frac{\partial}{\partial x}\left\{\frac{\sin \left(\Delta_{N} x / 2\right)}{\Delta_{N} \sin (x / 2)}\right\} .
$$

\section{c. Discrete delta-function approximations}

When $x$ is an integer, the function

$$
k_{N}(x)=\frac{\sin \left(\Delta_{N} x / 2\right)}{\Delta_{N} \sin (x / 2)}
$$

is a Kronecker delta function $\delta_{x, 0}$. Correspondingly, when $h_{N}(x)$ is used in a summation like

$$
F_{h}(x)=\sum_{x^{\prime}} h_{N}\left(x-x^{\prime}\right) F\left(x^{\prime}\right),
$$

provided $F\left(x^{\prime}\right)$ changes sufficiently smoothly as $x^{\prime}=$ $\ldots, x-2, x-1, x, x+1, x+2, \ldots$, then we can write

$$
F_{h}(x) \approx \frac{F(x-1)-F(x+1)}{2},
$$

and thus $h_{N}(x)$ behaves like a "derivative" of a Kronecker delta function. If $F(x)$ is smooth over a wide range of integer values of $x$, so that the range of $x$ can be regarded as a continuum, the finite difference (A18) becomes essentially the same as a derivative. This is the limit in which we shall use these functions. 


\section{d. Phase space behavior of the wavelets}

By using the WKB approximation, we can derive an approximate form for the wavelet functions in the form

$$
\begin{aligned}
w_{\bar{E}_{N}, \theta}(x) \approx & \frac{1}{\sqrt{p_{\bar{E}_{N}}(x) \Delta_{N}}} \exp \left(\frac{i}{\hbar} \int_{0}^{x} d x^{\prime} p_{\bar{E}_{N}}\left(x^{\prime}\right)\right) \\
& \times \frac{\sin \left\{\Delta_{N}\left[\theta+\theta\left(x, \bar{E}_{N}\right)\right] / 2\right\}}{\sin \left\{\left[\theta+\theta\left(x, \bar{E}_{N}\right)\right] / 2\right\}} .
\end{aligned}
$$

In this equation, the function

$$
p_{\bar{E}_{N}}(x)=\sqrt{2 M\left(\bar{E}_{N}-V(x)\right)}
$$

is the classical momentum of the atom at point $x$, while the function

$$
\theta\left(x, \bar{E}_{N}\right)=M \omega_{N} \int_{0}^{x} \frac{d x^{\prime}}{p_{\bar{E}_{N}}\left(x^{\prime}\right)}
$$

is proportional to the classical time taken for the atom to reach the point $x$; indeed if the trap is exactly harmonic with trap frequency $\omega$, then $\hbar \omega_{N}=\hbar \omega$, and $\theta\left(x, \bar{E}_{N}\right)$ is exactly the phase angle $\omega t$ of the oscillator at the time taken to reach the point $x$.

The approximate form of the wavelet function (A19) shows that it is sharply peaked when $\theta=-\theta\left(x, \bar{E}_{N}\right)$, so that the wavelets represent a localization at the point where a classical particle would have phase equal to $\theta\left(x, \bar{E}_{N}\right)$

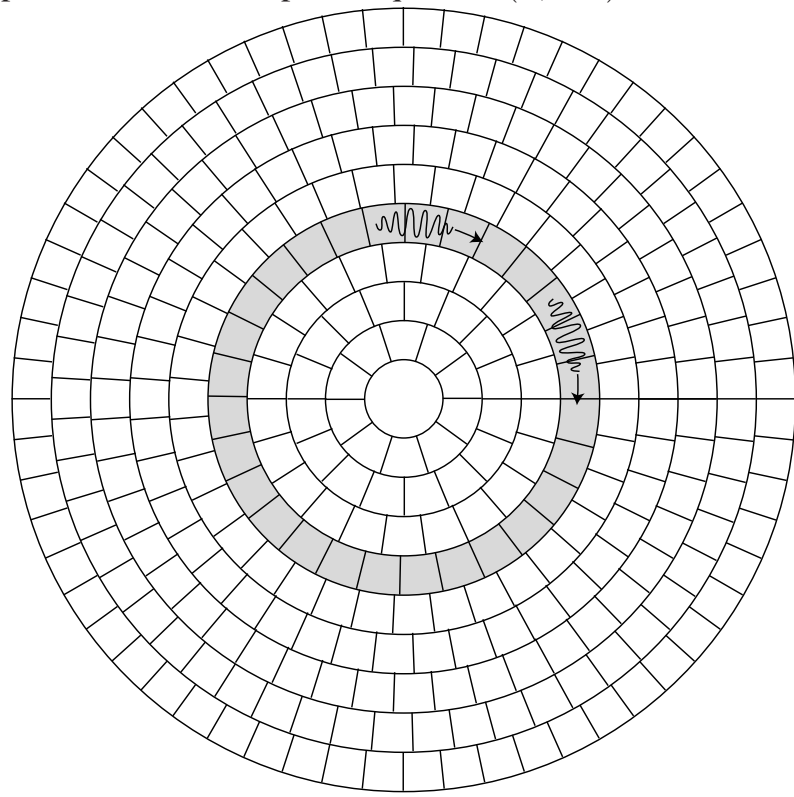

Fig.3. The phase space cells for a harmonic oscillator

\section{e. Harmonic trapping potential}

In the case of the harmonic oscillator potential $V(x)=$ $k x^{2} / 2$, we can label the wavelets by a momentum $p(N, \theta)$ and position $x(N, \theta)$ given by

$$
\begin{aligned}
& r(N, \theta)=\sqrt{2 \bar{E}_{N} / k} \cos \theta \\
& p(N, \theta)=\sqrt{2 M \bar{E}_{N}} \sin \theta
\end{aligned}
$$

and hence get a set of values of $r$ and $p$ which can be used to label the wavelets. This set is completely equivalent to the $N, \theta$ labelling.

\section{f. Size of the phase space cells}

The value of $\Delta_{N}$ is not yet fixed, and it should be chosen for the maximum convenience. The best choice is one which makes the phase space cells all have approximately the same dimensions. For large $N$, we will show that this can be achieved by the choice

$$
\begin{aligned}
\Delta_{N} & \approx \Delta \sqrt{\bar{E}_{N}}, \\
\Delta & =2 \sqrt{\frac{2 \pi}{\hbar \omega}} .
\end{aligned}
$$

With this choice, the uncertainties of the phase and energy variables are

$$
\begin{aligned}
\delta \bar{E}_{N} & =\hbar \omega \Delta \sqrt{\bar{E}_{N}} \\
\delta \theta_{N} & =\frac{2 \pi}{\Delta \sqrt{\bar{E}_{N}}}
\end{aligned}
$$

so that, using (A22, A23, the uncertainties in $r$ and $p$ are

$$
\begin{aligned}
& \delta r=|\sin \theta| \frac{2 \pi \sqrt{2}}{\Delta \sqrt{k}}+|\cos \theta| \frac{\hbar \omega \Delta}{2 \sqrt{2 k}} \\
& \delta p=|\cos \theta| \frac{2 \pi \sqrt{2 M}}{\Delta}+|\sin \theta| \frac{\hbar \omega \sqrt{M}}{2 \sqrt{2}} .
\end{aligned}
$$

Here $\delta r$ and $\delta p$ vary quite strongly with the phase $\theta$, unless the coefficients of the $\sin \theta$ and $\cos \theta$ in each of these equations are the same, and this happens when $\Delta=2 \sqrt{2 \pi / \hbar \omega}$, as in A25.

In this case the uncertainties become

$$
\begin{aligned}
& \delta r=\sqrt{\frac{\pi \hbar}{\eta}}(|\sin \theta|+|\cos \theta|) \\
& \delta p=\sqrt{\pi \hbar \eta}(|\sin \theta|+|\cos \theta|) .
\end{aligned}
$$

with $\eta=(k M)^{1 / 4}$.

The coefficients of $|\sin \theta|+|\cos \theta|$ are essentially the variances of $r$ and $p$ in the ground state of a harmonic oscillatorthus we find $h / 2 \leq \delta r \delta p \leq h$, which means that the cells correspond basically to minimum uncertainty states. The "cells" are thus square when measured in these natural units, and the variation in $\delta r$ and $\delta p$ arises solely because their orientations depend on $\theta$.

The choice A24) of $\Delta_{N}$ naturally leads to the the fact that for large $N$

$$
\bar{E}_{N} \approx \frac{\Delta^{2} N^{2}}{4}
$$




\section{One dimensional field Hamiltonian in terms of wavelets}

The field Hamiltonian can be written in terms of the noninteracting and the collisional parts

$$
H=H_{\text {kin }}+H_{\mathrm{T}}+H_{\mathrm{I}}
$$

We can write the non-interacting part as

$$
H_{\mathrm{kin}}+H_{\mathrm{T}}=\sum_{N \theta} \bar{E}_{N} a_{N \theta}^{\dagger} a_{N \theta}+\sum_{N \theta \theta^{\prime}} M_{N}\left(\theta, \theta^{\prime}\right) a_{N \theta}^{\dagger} a_{N \theta^{\prime}}
$$

Here $M_{N}\left(\theta-\theta^{\prime}\right)$ is defined in terms of the matrix elements A14, A15 as

$$
M_{N}\left(\theta-\theta^{\prime}\right)=-i \hbar \omega_{N} h_{N}\left(\theta-\theta^{\prime}\right) .
$$

\section{a. Phase space description}

If we use the labelling in terms of $p$ and $r$, the structure of the description is unaltered, but the appearance of the expression looks different. Thus we can write

$$
H_{\mathrm{kin}}+H_{\mathrm{T}}=\sum_{r p} \bar{E}_{r p} a_{r p}^{\dagger} a_{r p}+\sum_{r r^{\prime} p p^{\prime}} M\left(r r^{\prime} p p^{\prime}\right) a_{r p}^{\dagger} a_{r^{\prime} p^{\prime}} .
$$

This expression conceals the nature of the wavelet basis, which is implied only by the fact that the phase space points $r, p$ represent wavelets through the definition A22, A23, which arranges the points on discrete energy shells at positions on these shells determined by the values of $\theta$. The nature of the function $M\left(r r^{\prime} p p^{\prime}\right)$ means that it is nonzero only for points $r, p$ and $r^{\prime}, p^{\prime}$ which are on the same energy shell. When acting on sufficiently smooth functions we can use the approximate relations of sect.A 1d, and in particular (A18), $F(r, p)$, and thus approximate

$$
\sum_{r r^{\prime} p p^{\prime}} M\left(r r^{\prime} p p^{\prime}\right) F\left(r^{\prime}, p^{\prime}\right) \approx i \hbar\left(\frac{p}{M} \frac{\partial}{\partial r}-k r \frac{\partial}{\partial p}\right) F(r, p) .
$$

Thus the Liouvillian flow arises for sufficiently smooth functions, and we can formally write

$$
\left[H, a_{r p}\right] \approx \bar{E}_{r p} a_{r p}+i \hbar\left(\frac{p}{M} \frac{\partial}{\partial r}-k r \frac{\partial}{\partial p}\right) a_{r p}
$$

provided $a_{r p}$ is interpreted as being evaluated in an average over a density operator for a state which satisfies the smoothness requirement.

\section{Three dimensional formulation}

The case of a harmonic potential is particularly simple to treat, since the energies in the three different dimensions are separately conserved. We will use a notation in terms of these three energy components as follows. Each "cell" is specified by a set of six quantities $\left(N_{1}, N_{2}, N_{3}, \theta_{1}, \theta_{2}, \theta_{3}\right)$, from which we can form the two vector quantities $\mathbf{r}, \mathbf{Q}$, defined by

$$
\begin{aligned}
r_{i} & =\sqrt{\frac{2 \bar{E}_{N, i}}{k}} \cos \theta_{i}, \\
\hbar Q_{i} & =\sqrt{2 M \bar{E}_{N, i}} \operatorname{sign}\left(\sin \theta_{i}\right) .
\end{aligned}
$$

The definition of $\mathbf{Q}$ ensures that the set $\mathbf{r}, \mathbf{Q}$ do determine $\left(N_{1}, N_{2}, N_{3}, \theta_{1}, \theta_{2}, \theta_{3}\right)$ uniquely; the sign of $Q_{i}$ determines the sign of the $i$-component of the momentum, since for a given $E_{i}$ and $r_{i}$ this can have two possible directions. In terms of these variables, we now use the notation $w_{\mathbf{Q}}(\mathbf{x}, \mathbf{r})$ for the wavelet function, and the notation $A_{\mathrm{Q}, \mathbf{r}}$ for the corresponding destruction operator, so that the field operator can be expressed as

$$
\begin{aligned}
\psi(\mathbf{x}) & =\sum_{\mathbf{Q}, \mathbf{r}} w_{\mathbf{Q}}(\mathbf{x}, \mathbf{r}) A_{\mathbf{Q}, \mathbf{r}} \\
& =\sum_{\mathbf{Q}} \psi_{\mathbf{Q}}(\mathbf{x})
\end{aligned}
$$

The commutation relations are of course

$$
\left[A_{\mathbf{Q}, \mathbf{r}}, A_{\mathbf{Q}^{\prime}, \mathbf{r}^{\prime}}^{\dagger}\right]=\delta_{\mathbf{r} \mathbf{r}^{\prime}} \delta_{\mathbf{Q Q}^{\prime}}
$$

The commutation relations for the $\psi_{\mathbf{Q}}(\mathbf{x})$ will be needed; they are

$$
\begin{gathered}
{\left[\psi_{\mathbf{Q}}(\mathbf{x}), \psi_{\mathbf{Q}^{\prime}}^{\dagger}\left(\mathbf{x}^{\prime}\right)\right]=\delta_{\mathbf{Q Q}^{\prime}} \sum_{\mathbf{r}} w_{\mathbf{Q}}^{*}(\mathbf{x}, \mathbf{r}) w_{\mathbf{Q}}\left(\mathbf{x}^{\prime}, \mathbf{r}\right)} \\
\approx \delta_{\mathbf{Q}, \mathbf{Q}^{\prime}} e^{i \mathbf{K}\left(\mathbf{Q}, \frac{\mathbf{x}+\mathbf{x}^{\prime}}{2}\right) \cdot\left(\mathbf{x}-\mathbf{x}^{\prime}\right)} g_{\mathbf{Q}}\left(\mathbf{x}, \mathbf{x}^{\prime}\right) .
\end{gathered}
$$

The approximation to get the second line comes from the WKB form, A19.

\section{a. General potentials}

The construction here is specific to a potentials which can be written as the sum of $x, y$ and $z$ dependent parts, of which the three-dimensional harmonic potential is the most useful example. Even though harmonic traps are most commonly used, the effect of the condensate on the other particles acts like an additional potential, and it will therefore be necessary for us to consider more general potentials. We will, without proof, assume that it is always possible to find an appropriate wavelet basis of the kind set up here. Although this may not be so, particularly in the case of non-integrable systems, it seems very likely that the results of our analysis will continue to be true. we say this because our methodology really depends only on a local properties, and almost every potential we are likely to use is locally harmonic. 


\section{APPENDIX B: TIME DEPENDENT MEAN-FIELD TERMS}

We will show that, under the requirement that that the momentum disrtibution function is smooth, we may use the approximate expression

$$
\begin{aligned}
\left\langle\psi_{\mathbf{Q}}^{\dagger}(\mathbf{x}) \psi_{\mathbf{Q}}^{\dagger}\left(\mathbf{x}^{\prime}\right)\right\rangle= & e^{i \mathbf{K}(\mathbf{Q}, \mathbf{u}) \cdot \mathbf{v}}\left\{g\left(\mathbf{x}, \mathbf{x}^{\prime}\right) F(\mathbf{K}(\mathbf{Q}, \mathbf{u}), \mathbf{u})\right. \\
& \left.-i\left(\nabla_{\mathbf{v}} g\left(\mathbf{x}, \mathbf{x}^{\prime}\right)\right) \cdot \nabla_{\mathbf{K}} F(\mathbf{K}(\mathbf{Q}, \mathbf{u}), \mathbf{u})\right\}
\end{aligned}
$$

in which $\mathbf{x}=\mathbf{u}+\mathbf{v}$ and $\mathbf{x}^{\prime}=\mathbf{u}-\mathbf{v}$. We will do this explicitly for the case of no trapping potential as follows. In this case we consider

$$
\begin{aligned}
& \left\langle\psi_{\mathbf{K}}^{\dagger}(\mathbf{x}) \psi_{\mathbf{K}}^{\dagger}\left(\mathbf{x}^{\prime}\right)\right\rangle \\
& \quad=\frac{1}{(2 \pi)^{3}} \iint_{\mathbf{K}-\boldsymbol{\Delta}}^{\mathbf{K}+\boldsymbol{\Delta}} d^{3} \mathbf{k} d^{3} \mathbf{k}^{\prime}\left\langle a_{\mathbf{k}}^{\dagger} a_{\mathbf{k}^{\prime}}\right\rangle e^{i\left(\mathbf{k} \cdot \mathbf{x}-\mathbf{k}^{\prime} \cdot \mathbf{x}^{\prime}\right)}
\end{aligned}
$$

We can now express the smoothness requirement as the condition that the mean $\left\langle a_{\mathbf{k}}^{\dagger} a_{\mathbf{k}^{\prime}}\right\rangle$ can be expressed as a function $R(\mathbf{p}, \mathbf{q})$ of $\mathbf{p}=\left(\mathbf{k}+\mathbf{k}^{\prime}\right) / 2$ and $\mathbf{q}=\mathbf{k}-\mathbf{k}^{\prime}$. The requirement that the different bands in $\mathbf{K}$ be uncorrelated, which is fundamental to the method, only makes physical sense if any correlation that does exist between $\mathbf{k}$ and $\mathbf{k}^{\prime}$ is over a range in $\mathrm{k}$ that is very much less than $\Delta$, the spacing between the bands. Thus $R(\mathbf{p}, \mathbf{q})$ must be sharply peaked in $\mathbf{q}$. On the other hand, the dependence of $R(\mathbf{p}, \mathbf{q})$ on $\mathbf{p}$ represents the overall momentum distribution, which should be smooth over the range $\Delta$ between the bands.

This means that we can change to the variables $\mathbf{p}, \mathbf{q}$ in the integral, and to a good approximation let the limits of the $\mathbf{q}$ integration go to infinity, while those of the $\mathbf{p}$ are $\mathbf{K}-\boldsymbol{\Delta}, \mathbf{K}+\boldsymbol{\Delta}$. We implement the smoothness in $\mathbf{p}$ requirement by using a Taylor expansion for $R(\mathbf{p}, \mathbf{p})$, getting first order approximation

$$
\begin{aligned}
& \left\langle\psi_{\mathbf{K}}^{\dagger}(\mathbf{x}) \psi_{\mathbf{K}}^{\dagger}\left(\mathbf{x}^{\prime}\right)\right\rangle \\
& \approx \frac{1}{(2 \pi)^{3}} \int_{-\infty}^{\infty} d^{3} \mathbf{q} \int_{\mathbf{K}-\Delta}^{\mathbf{K}+\boldsymbol{\Delta}} d^{3} \mathbf{p} e^{-i(\mathbf{p} \cdot \mathbf{u}+\mathbf{q} \cdot \mathbf{v})} \\
& \times\left\{R(\mathbf{K}, \mathbf{q})(\mathbf{p}-\mathbf{K}) \cdot \nabla_{\mathbf{K}} R(\mathbf{K}, \mathbf{q})\right\}, \\
& =e^{i \mathbf{K} \cdot \mathbf{v}}\left\{g\left(\mathbf{x}, \mathbf{x}^{\prime}\right) F(\mathbf{K}, \mathbf{u})-i\left(\nabla_{\mathbf{v}} g\left(\mathbf{x}, \mathbf{x}^{\prime}\right)\right) \cdot \nabla_{\mathbf{K}} F(\mathbf{K}, \mathbf{u})\right\}
\end{aligned}
$$

where in this case

$$
g\left(\mathbf{x}, \mathbf{x}^{\prime}\right)=\frac{\sin \Delta_{x}\left(x-x^{\prime}\right)}{\pi\left(x-x^{\prime}\right)} \frac{\sin \Delta_{y}\left(y-y^{\prime}\right)}{\pi\left(y-y^{\prime}\right)} \frac{\sin \Delta_{z}\left(z-z^{\prime}\right)}{\pi\left(z \cdot-z^{\prime}\right)},
$$

as in QKI.

Using the formula (B3), tjhe commutation relations $2.12,2.12$ ) and the Gaussian factorization of the correlation functions, it is not difficult to derive the form of the correction 6.5). It should be noted that the smoothness assumption will not be valid at the edge of the non-condensate band, where a boundary layer will form. This results from the fact that the Liouvillian straming in the time-dependent potential is not parallel to the actual fixed boundary between the two bands.

[1] M. Anderson, J.R. Ensher, M.R. Matthews, C.E. Wieman and E.A. Cornell, Science 269, 198 (1995).

[2] C.C. Bradley, C.A. Sackett, J.J. Tollet, and R. Hulet, Phys. Rev. Lett. 75, 1687 (1995).

[3] K. B. Davis , M.-O. Mewes , M. R. Andrews , N. J. van Druten, D. S. Durfee, D. M. Kurn, W. Ketterle, Phys. Rev. Lett. 75, 3969 (1995);

[4] For a current lising of condensates, see the BEC Home Page, http://amo.phy.gasou.edu/bec.html/

[5] N.N. Bogoliubov, J.Phys. (USSR), 11, 23, (1947); reprinted in D. Pines, The Many Body Problem, Benjamin N.Y. (1962);

[6] A. L. Fetter, Ann. Phys. (N.Y.) 70, 67, (1972); A. L. Fetter, Phys. Rev. A 53, 4246 (1996); M. Lewenstein and L. You, Phys. Rev. A 53, 909 (1996); L. You, W. Hoston and M. Lewenstein, Phys. Rev. A 55, R1581 (1997); M. Lewenstein, L. You, Phys. Rev. Lett. 77, 3489, (1996)

[7] C.W. Gardiner, Phys. Rev. A 56, 1414 (1997); R. Dum, Y. Castin, Physical Review A 57, 3008 (1998).

[8] V.L. Ginzburg and L.P. Pitaevskii, Zh. Eksp. Teor.Fiz. 34, 1240 (1958) [Sov. Phys. JETP 7, 858 (1958)]; E.P. Gross, J. Math.Phys. 4, 195 (1963)

[9] A.A. Abrikosov, L.P. Gorkov and I.E. Dzyaloshinski, Methods of Quantum Field Theory in Statistical Physics, Dover NY (1963); E.M. Lifshitz and L.P. Pitaevskii, Statistical Physics Part 2, Landau and Lifshitz Course of Theoretical Physics Vol. 9 (Pergamon Press, Oxford 1980)

[10] A. Griffin, Excitations in a Bose-Condensed Liquid, Cambridge University Press, (Cambridge 1993)

[11] In this paper will use the notation: QKI for [12], QKII for [13, QKIII for [14], and QKIV for [15]

[12] C.W. Gardiner and P. Zoller, Phys. Rev. A 55, 2902 (1997)

[13] D. Jaksch, C.W. Gardiner and P. Zoller, Phys. Rev. A 56, 575 (1997)

[14] C.W. Gardiner and P. Zoller, Phys. Rev. A 58, 536 (1998)

[15] D. Jaksch, C.W. Gardiner and P. Zoller, Phys. Rev. A 58, 1450 (1997)

[16] C.W. Gardiner, P. Zoller, R.J. Ballagh and M.J. Davis, Phys. Rev. Lett. 79, 1793 (1997).

[17] C.W. Gardiner, M.D. Lee, R.J. Ballagh, M.J. Davis and P. Zoller, Phys. Rev. Lett. 81, 5266 (1998).

[18] L.W. Nordheim, Proc. Roy. Soc. A, 119, 689 (1928); F. Bloch, Z. Phys. 52, 555, (1928); S. Kikuchi and L. Nordheim, Z. Phys. 60, 652 (1930); E. A. Uehling and G.E. Uhlenbeck, Phys. Rev. 43, 552 (1933).

[19] E. Levich and V. Yakhot, Phys. Rev. B 15, 243 (1977); J.Phys.A 11, 2237 (1978); D.W. Snoke and J.P. Wolfe, Phys. Rev. B 39, 4030 (1989); B. Svistunov, J. Mosc. Phys. Soc. 1, 373 (1991); H.T.C. Stoof, Phys. Rev. Lett. 66, 3148 (1991); Yu. M. Ka- 
gan, B. V. Svistunov and G.V. Shlyapnikov, Sov. Phys JETP 75, 387 (1992); D.V. Semikoz and I.I. Tkachev, Phys. Rev. Lett. 74, 3093 (1995); H.T.C. Stoof, Phys. Rev. A 49, 3824 (1994); BoseEinstein Condensation, edited by A. Griffin, D. W. Snoke, and S. Stringari (Cambridge University Press, Cambridge, 1995).

[20] A. Griffin, Physical Review B 53, 9341 (1996). This paper summarizes the various "standard" ways of using the Hartree-FockBogoliubov method, and discusses the problems that can occur. See also [6,24,25] and S. A. Morgan, S. Choi and K. Burnett, Phys. Rev. A 57, 3818 (1998)

[21] H. T. C. Stoof, J. Low Temp. Phys. 114, 11 (1999)

[22] R. Walser, J. Williams, J. Cooper, and M. Holland Phys. Rev. A 59, 3878 (1999)

[23] E. Zaremba, A. Griffin and T. Nikuni, Phys. Rev. A 57, 4695 (1998)
[24] N. P. Proukakis, K. Burnett and H. T. C. Stoof Phys. Rev. A 57, 1230 (1998) gives an excellent exposition of the issues which must be addressed in this approximation.

[25] F. Dalfovo, S. Giorgini, L. P. Pitaevskii and S. Stringari, Rev. Mod. Phys. 71, 463 (1999)

[26] L. You, W. Hoston and M. Lewenstein, Phys. Rev. A 55, R1581 (1997) present and solve these equations in the form most appropriate to current condensates.

[27] R. Graham, Phys. Rev. Lett. 81, 5262 (1998)

[28] P. D. Drummond and J. F. Corney, Quantum Dynamics of Evaporatively Cooled Bose-Einstein Condensates, cond-mat/9806315, (1998)

[29] M. Girardeau and R. Arnowitt, Phys. Rev. 113, 755 (1959); M. Girardeau, Phys. Rev. A 58, 775 (1998) 


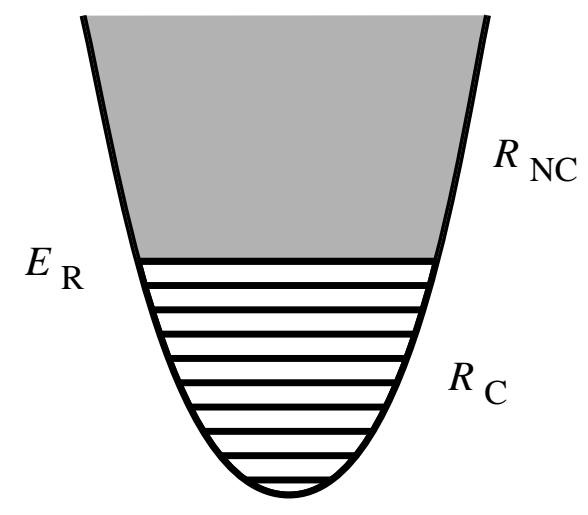

